Nouvelles perspectives en sciences sociales

\title{
Rites funèbres et sciences humaines : synthèse et hypothèses
}

\section{Luc Bussières}

Volume 3, numéro 1, septembre 2007

URI : https://id.erudit.org/iderudit/602466ar

DOI : https://doi.org/10.7202/602466ar

Aller au sommaire du numéro

Éditeur(s)

Prise de parole

\section{ISSN}

1712-8307 (imprimé)

1918-7475 (numérique)

Découvrir la revue

\section{Citer cet article}

Bussières, L. (2007). Rites funèbres et sciences humaines : synthèse et hypothèses. Nouvelles perspectives en sciences sociales, 3(1), 61-139. https://doi.org/10.7202/602466ar

\section{Résumé de l'article}

Les rites funèbres sont des rites de passage vieux de 100 millénaires. Ils ont différentes fonctions sociales, dont celle de se placer entre la mort et nous, entre la mort et la culture. Plusieurs auteurs de diverses sciences humaines ont écrit à propos des modifications récentes et profondes du monde de la ritualité funéraire en Occident, soit pour parler de déritualisation, soit pour parler d'invention de "nouveaux rituels ». Nous proposons ici une synthèse des savoirs sur la place des rites funèbres fondée par l'observation des sociétés de la tradition et de la modernité, de même qu'une série d'hypothèses, à vérifier empiriquement, en contexte de postmodernité. 


\title{
Rites funèbres et sciences humaines : synthèse et hypothèses
}

\author{
LUC BUSSIËRES \\ Université de Hearst
}

"La mort est une découverte récente et inachevée ${ }^{1}$ "

La mort a connu un traitement inégal dans l'histoire des sciences humaines et sociales. Tour à tour absente, sujet tabou, sujet jugé hors d'atteinte de nos modes de connaissance ou sujet d'intenses débats au cœur de nos disciplines. Mais si inégal qu'ait été ce traitement, la mort a toujours été à l'horizon du discours sur la société, quand elle n'en a pas été tout simplement pur objet. Si inégal qu'ait été le traitement, les sciences sociales contemporaines n'ont pas manqué de constater que la mort prenait des visages à la fois semblables et différents tout au long de l'histoire de l'humanité et que, fort probablement, la postmodernité l'aurait peut-être un peu transformée.

Les adeptes et praticiens des sciences de l'humain sont devant un défi important face à l'interprétation à donner au rôle de la mort dans l'histoire et à l'évolution de ce rôle jusqu'à aujourd'hui. La mort a toujours constitué pour l'humain un sujet important de réflexion. Pourtant dans la modernité, les sciences de l'humain n'y ont accordé un intérêt réel qu'à partir des publications d'Edgar Morin, au début des années cinquante, de Geoffrey Gorer dans les années soixante et, dans les années soixante-dix, avec les Philippe Ariès, Louis-Vincent Thomas,

1 André Malraux, Lazare, Paris, Gallimard, 1974, p. 241. 
Jean Ziegler, Michel Vovelle et Jean Baudrillard. Cette première génération de travaux en anthropologie, en histoire et en sociologie sur le thème de la mort a ensuite donné lieu à un foisonnement d'écrits et à un élargissement des perspectives. On a même suggéré le regroupement des études sur la mort autour d'une nouvelle science, la thanatologie. Peu à peu, la mort s'est constituée en objet de recherche, publications et colloques sur ce thème devenant de plus en plus courants, ce qui a favorisé l'émergence d'une toute nouvelle génération de penseurs et de chercheurs qui ont donné suite à la première.

À nos yeux, ces travaux appellent une synthèse. C'est en cela que se veut notre première contribution. Cette synthèse révélera que les analyses si pertinentes, si percutantes soient-elles sont souvent menées en contournant les impératifs d'un travail de vérification empirique. Ces impératifs sont contournés, nous semble-t-il, parce que les questionnements ne sont pas modelés de telle manière qu'ils donnent lieu à des hypothèses. C'est dans la constitution de ces hypothèses que se situe notre seconde contribution. Plus particulièrement, nous voudrons mettre en relation l'évolution de nos pratiques funéraires et, indirectement, de notre rapport à la mort avec le contexte culturel typique de la postmodernité occidentale, l'essentiel du travail de description et d'analyse sur ces sujets ayant été élaboré en étudiant et en comparant les sociétés de la tradition et celles de la modernité.

\section{Prolégomènes à une réflexion contemporaine sur la mort}

\subsection{Aux sources de l'aventure humaine : « la mort provoque la culture $^{2} »$}

Assurer la survie ou la vie n'a jamais été une sinécure pour nos ancêtres de la préhistoire ni d'ailleurs pour ceux de l'ensemble des sociétés préindustrielles, mais, dès le moment où la conscience de notre mort leur est apparue, quelque chose d'important venait d'être bousculé pour de bon. Notre nature animale avait répondu jusque là à trois instincts

2 Selon l'expression de Patrick Baudry dans "Paradoxes contemporains. Nouveaux rapports anthropologiques à la mort ", dans Frédéric Lenoir et Jean-Philippe de Tonnac (dir.), La mort et l'immortalité. Encyclopédie des savoirs et des croyances, Paris, Bayard, 2004, p. 895 . 
fondamentaux ${ }^{3}$ : la survie physique par la recherche de nourriture, l'autodéfense contre les agressions et la reproduction de l'espèce. S'ajoutait alors nécessairement une difficulté supplémentaire, une autre question à régler : une question dite existentielle et incontournable. Comment, en effet, redéfinir la vie elle-même dans ce nouveau contexte? Qu'allait-on faire dorénavant de notre « encombrant savoir » sur la mort, comme l'a admirablement formulé Jean-Didier Urbain ${ }^{4}$ ? C'est ce que Michel Hulin, rappelle ainsi :

Aucune évidence n'est plus écrasante que la mort. [...] au point que la pratique de l'inhumation, la seule à laisser des traces durables, passe aux yeux de beaucoup - plus que l'outil et le langage, dont il existe des ébauches animales - pour le signe même de l'avènement de la condition humaine en tant que telle.

Reste à comprendre comment cette protestation symbolique initiale, en apparence vaine et mème dérisoire, a pu se prolonger et s'amplifier à travers les millénaires, entraînant dans son sillage la naissance des religions et des civilisations elles-mêmes, pour déboucher finalement sur le rapport problématique, fait de déni et de fascination hébétée, qu'entretient avec la mort l'homme d'aujourd'hui'.

[...] C'est sans doute à cette double absurdité de l'inconcevable et de l'inadmissible que s'est heurté le premier hominidé qui, rompant avec l'indifférence animale en face des cadavres, s'est demandé où se trouvait désormais cet être cher qui venait d'expirer dans ses bras. Qu'est-ce qui lui a alors permis de surmonter son accablement et de poser, en un défi lancé à la mort, les premiers soubassements de ce qui deviendrait au fil des siècles le majestueux édifice des rites funéraires et des mythes eschatologiques'?

On atteint ici l'ordre de la culture. C'est probablement pour cela qu'on a pu dire que «la sépulture crée la culture ${ }^{7}$ ou que Marcel Mauss a pu affirmer que c'est la mort qui a appris aux humains à parler.

Emmanuel Anati, La religion des origines, Paris, Bayard, 1999, p. 27.

4 Jean-Didier Urbain, L'arbipel des morts. Le sentiment de la mort et les dérives de la mémoire dans les cimetieres d'Occident, Paris, Éditions Payot et Rivages, [1989] 1998, p. 24.

" Michel Hulin, «L'homme et son double ", dans Frédéric Lenoir et Jean-Philippe de Tonnac (dir.), La mort et l'immortalité. Encyclopédie de la mort et des croyances, op. cit., p. 55.

Ibid., p. 57.

Éric Volant, “La religion et la mort», dans Jean-Marc Larouche et Guy Ménard (dir.), L’étude de la religion au Québec. Bilan et prospective, Québec, Les Presses de l'Université Laval/Corporation canadienne des sciences religieuses, 2001, p. 323. 
Dans l'état actuel de nos connaissances, tout laisse croire que ce moment décisif de notre histoire, l'invention de la sépulture, remonte à environ 100000 ans, alors qu'Homo Neandertalensis et Homo sapiens cohabitaient sur la terre ${ }^{8}$. Les deux espèces ou deux lignées humaines ont développé des pratiques funéraires d'inhumation, les plus anciennes que l'on connaisse'.

Cette préoccupation supplémentaire que nos ancêtres venaient de s'imposer - celle de s'arrêter pour prendre soin d'un cadavre, pour lui trouver un lieu offrant une protection adéquate contre les animaux et les éléments, pour le parer, pour réunir les offrandes (bijoux, nourriture, ustensiles, vêtements, armes, fleurs, etc.) qu'on donnait à la personne défunte (s'en privant du même coup) et qui très tôt ont accompagné les premières sépultures pour se généraliser par la suite, il y a environ $40000 \mathrm{ans}^{10}-$, tout cela doit trouver sa justification quelque part. Pourquoi se donner ainsi tant de mal alors même que la survie quotidienne est tellement aléatoire? Quand ces premières communautés humaines, ces premières organisations sociales, ont été confrontées à ce nouveau problème, que faire de nos morts, il a fallu trouver une solution pour que survive au moins le groupe. Et c'est le monde des idées qui pouvait offrir des pistes de solution puisque biologiquement et matériellement la partie était perdue d'avance pour l'individu et que, d'entrée de jeu, cette perspective n'apparaissait ni envisageable ni acceptable. Pour reprendre une expression d'Éric Volant, il a fallu apprendre à « ruser avec la mort ${ }^{11}$ ». Les réponses que l'on allait trouver à la question de l'après-mort seraient déterminantes pour le quotidien de ces premières communautés comme pour l'avenir de l'espèce. En effet, le désordre fondamental qu'introduit l'irruption de la mort dans la vie des humains et des groupes qu'ils forment ne peut rester sans

* Jean-Pierre Mohen, Lepropre de l'espèce humaine. Rites funéraires et destination du cadavre dans la prébistoire et les traditions anciennes, dans Frédéric Lenoir et Jean-Philippe de Tonnac (dir.), op. cit., p. 288-290.

"Si des crémations ont ćté pratiquées à cette époque ou antérieurement, il ne nous est pas possible, on le comprendra, d'en retrouver les traces, attestant ainsi de preuves de rites funéraires potentiellement encore plus anciens.

(1) Jean-François Dortier, L'bomme, cet étrange animal... Aux origines du langage, de la culture et de la pensée, Auxerre, Sciences Humaines Éditions, 2004, p. 270.

Éric Volant, Jeux mortels et enjeux éthiques, Chicoutimi, Sapienta, 1992, p. 27-60. 
réplique organisée et collective. Comme l'a formulé si justement Patrick Baudry, « la mort provoque la culture ». Qu'a-t-on donc trouvé dans le monde des idées pour réagir et pour ruser avec la « mortanéantissement "?

\subsection{Contre l'angoisse de la mort, les ruses de la pensée : les rites, le} sacré, les mythes, le langage et la religion

«L'attitude rituelle à l'égard du mort ne semble pas découler d'une logique rationnelle basée sur les trois instincts fondamentaux que sont la recherche de la nourriture, l'autodéfense et la reproduction de l'espèce ${ }^{12} »$. Tout laisse croire que ce questionnement initial sur le sens et la portée de la mort ait correspondu à l'évolution sensible de nos capacités intellectuelles sur le plan de l'abstraction et à la mise en place du culte des morts pour la première fois avec l'homme de Neandertal ${ }^{13}$. Remarquons que l'attitude rituelle devant la mort se développe au départ en dehors de tout cadre religieux ou institutionnel. En cela, et il est important de le noter pour la suite de notre propos, la pratique rituelle a dès le départ quelque chose de sauvage, de primitif ou d'indompté. Le rite prend son origine dans la rencontre avec le sacré.

De façon synthétique, on pourrait dire que, avec le développement de la pensée symbolique ${ }^{14}$, nos ancêtres se questionnent à propos de la mort et du sens à donner à leur existence ; ils pratiquent dès lors l'inhumation de leurs morts. Ils font l'expérience de la rencontre avec le sacré. Pour répondre à un besoin de plus en plus pressant de comprendre, ils développent, grâce au langage et aux symboles, nos premiers systèmes explicatifs, les mythes. Si le mythe est à l'arrièrescène, le rite vibre au quotidien et les systèmes religieux assumeront par la suite la mise en scène nécessaire à la reproduction et à la transmission de toute cette mécanique structurante, constituant ainsi notre réponse

Emmanuel Anati, op. cit., p. 53.

Ibid., p. 46.

14 Cette période correspond aussi à l'accélération, au paléolithique supérieur, du développement de l'expression artistique chez nos ancêtres, particulièrement avec l'art rupestre (peinture sur les parois rocheuses des cavernes), l'art mobilier (sculptures de statuettes et de colliers) et la musique. 
collective à la mort. Cette mort qui voulait tout emporter de l'humain mortel, lui qui se présente et se définit dorénavant comme un humain immortel. Un seuil historique et culturel fondamental vient d'être franchi. Jean-Didier Urbain résume très bien ce qu'il y a d'universel dans la rencontre de l'humain avec la mort et dans la construction des mythes et des rites qui en a résulté sur un mode relativement invariant comme on l'a vu:

L'homme de toutes les cultures s'est toujours heurté à cet inéluctable phénomène, à la répétition tragique de ce spectacle. [...] Devoir jouer cette scène est la première des certitudes. Mais l'hom me n'a pu admettre qu'elle soit aussi la dernière : celle de l'anéantissement de soi. Il n'a pu que croise que cet événement obligé devait être autre chose, signe d'autre cbose. L'homme a alors tendu au-dessus de ce "trou aveugle, absolument ${ }^{1.5}$ », un filet de mythologies et de rites apportant à sa conviction une essentielle cohérence. Ce filet est fragile mais sans cesse remaillé par la croyance, resserré lorsque le doute menace, au besoin remplacé par un autre au terme de ces déchirures qui ponctuent l'histoire des mentalités [...].

Il s'agit en quelque sorte de circonvenir la mort, de la séduire, d'obtenir d'elle qu'elle se dépouille de son absurdité primitive et brutale. Et c'est pour ce faire que l'homme a créé des récits et inventé des gestes, produit des discours et institué des pratiques, élaboré des paroles justifiantes et développé des techniques rassurantes qui, socialisant ses convictions, leur ont donné force de loi. 11 a ainsi mis en place, fabriqué peu à peu, toute une panoplie de procédés symboliques efficaces transformant ses rêveries en langage et sublimant son complexe $\mathrm{d}^{\prime} \mathrm{Orphée}{ }^{16}$ en théorie positive. La finalité de cette entreprise, ce n'est pas d'abolir la mort, de la nier ou de la cacher, mais de la transfigurer, de la traduire, de lui donner un sens : une raison d'être, une utilité, un avenir, une valeur, des qualités enfin permettant de passer outre la cruauté aveugle de l'inéluctable.

De la sorte, si la mort vécue que l'on observe dans la vie sociale est objectivement une série de gestes et de rites qui va de l'ultime maladie au tombeau et au-delà, elle est surtout le reflet de rêveries ordonnées qui ont suscité la mise en place d'un cadre sécurisant de pratiques funéraires - ces multiples pratiques "qui ont tenté en tout temps, d'apprivoiser la mort" ${ }^{17}$ ». Mais il ne peut s'agir alors d'instaurer une illusion de rapatriement des

Edgar Morin, L'bomme et la mort, Paris, Le Seuil, [1951] 1970, p. 101.

16 «Le complexe d'Orphée » est le titre d'un chapitre du livre de Jean-Didier Urbain (op. cit., p. 21-33) où il fait référence au fait que, en dehors de ce personnage de la mythologie grecque, personne n'a eu le loisir d'un aller et retour entre la vie et la mort.

1. Michel Vovelle, La mort et l'Occident de 1300 à nos jours, Paris, Gallimard, 1983, p. 9. 
morts dans le monde des vivants: Orphée lui-méme échoue dans cette tentative (Eurydice restera à jamais dans le monde des morts). Il s'agit de "domestiquer» [...] la diffúrence entre morts et vivants : non pas nécessairement de dissiper l'écart qui sépare ces deux mondes, ni même de réduire la distance entre eux ; mais seulement d'établir, d'abord leur cocxistence; et troubler, ensuite, en quelque manière, la radicalité de leur séparation. Non pas nier la frontière, mais rendre la limite relative ct propice à l'échange ${ }^{18}$.

\section{Recension des écrits sur la mort et les rites}

\section{1. «Au commencement était le rite ${ }^{19}$ »}

Les sciences humaines et sociales étudient les rites depuis une centaine d'années. De nombreux auteurs leur ont consacré une partie de leurs travaux (et parmi eux Claude Rivière ${ }^{20}$ qui mentionnera les contributions entre autres de Durkheim, Mauss, Van Gennep, Malinowski, RadcliffeBrown, Goffman, Turner, Lévi-Strauss, de même que celles de Girard, Freud, Huxley, Douglas, Caillois et Cazeneuve). Ces nombreuses études, par contre, bien qu'elles aient connu des convergences, n'ont pas été menées à partir d'un pur consensus sur le sens à donner au rite, pas plus qu'elles ont abouti à une entente généralisée.

Pour s'en rendre compte, on peut s'arrêter sur quelques citations et, d'abord, sur celle de Claude Rivière :

[...] les rites sont toujours à considérer comme ensembles de conduites individuelles ou collectives, relativement codifiées, ayant un support corporel (verbal, gestuel, postural), à caractère plus ou moins répétitif, à forte charge symbolique pour leurs acteurs et habituellement pour leurs témoins, fondées sur une adhésion mentale, éventuellement non conscientisée, à des valeurs relatives à des choix sociaux jugés importants, et dont l'efficacité attendue ne relève pas d'une logique purement

18 Jean-Didier Urbain, op. cit., p. 24-25.

19 Titre repris d'un ouvrage récemment traduit et publié du livre posthume d'Arthur Maurice Hocart (1883-1939) paru à l'origine en anglais en 1954 sous le titre Social Origins. Pour la version française actuelle, Au commencement était le rite. De l'origine des sociétés bumaines, Paris, Éditions I.a Découverte, 2005.

21) Claude Rivière, Les rites profanes, Paris, PUF, 1995, p. 21-45. 
empirique qui s'épuiserait dans l'instrumentalisation technique du lien cause-effet ${ }^{21}$.

D'autres citations permettent de constater à la fois des récurrences et des divergences. Dans les passages qui suivent, on lira en gras des éléments de définition ou des nuances qui n'apparaissent pas déjà dans le propos de Rivière.

Passons à celle de Martine Segalen :

Le rite ou rituel est un ensemble d'actes formalisés, expressifs, porteurs d'une dimension symbolique. Le rite est caractérisé par une configuration spatio-temporelle spécifique, par le recours à une série d'objets, par des systèmes de comportements et de langages spécifiques, par des signes emblématiques dont le sens codé constitue l'un des biens d'un groupe ${ }^{22}$.

Plus loin elle ajoute : «Finalement, le rituel se reconnaît à ce qu'il est le fruit d'un apprentissage, il implique donc la continuité des générations, des groupes d'âge ou des groupes sociaux au sein desquels ceux-ci se produisent ${ }^{23}{ }^{2}$.

Pascal Lardellier, pour sa part, le définit comme :

[...] un contexte social particulier, instauré au sein d'un dispositif de nature spectaculaire, caractérisé par son formalisme, et un ensemble de pratiques normatives, possédant une forte valeur symbolique pour ses acteurs et ses spectateurs.

Spectacle, donc, mais plus que cela, le rite est une performance, efficace symboliquement d'un point de vue social et institutionnel ${ }^{24}$.

Roy A. Rappaport précise quant à lui que : "I take the term ritual to denote the performance of more or less invariant sequences of formal acts and utterances not entirely encoded by the performers ${ }^{25}$ ».

Pour Denis Jeffrey :

D'une façon générale le rite donne à vivre des symboles passablement codifiés par une culture qui ont du sens pour un individu ou une collectivité. Ils sont relatifs à des moments de vie qui rappellent aux hommes leur jardin intérieur, leur identité et leurs conduites vis-à-vis des

Ibid., p. 11.

Martine Segalen, Rites et rituels contemporains, Paris, Nathan, 1998, p. 20.

Ibid., p. 21.

2. Pascal Lardellier, Théorie du lien rituel. Antbropologie et communication, Paris, L'Harmattan, 2003, p. $21-22$.

15. Roy A. Rappaport, Ritual and religion in the making of bumanity, Cambridge, Cambridge University Press, 1999, p. 24. 
forces qui les débordent. C'est pourquoi ils sont souvent teintés de mystère. I.e rite se répète au besoin et trouve son efficacité dans une logique symbolique fondée sur ce qu'elle rapporte en termes existentiels : apaisement, assurance, protection, paix de l'esprit, sécurisation, participation, libération, remémoration, purification intérieure, guérison, autorisation, passage, maîtrise de soi, transformation, différenciation, reconnaissance, identification, reliance, appartenance à un groupe, surplus d'énergie, enchantement, etc. ${ }^{20}$

Finalement Michel Hanus affirme que :

Le rite est un acte ou, plus souvent, un ensemble, une suite d'actes, de comportements, de conduites. S'il intéresse toute la personne qui y participe, celle-ci s'y trouve surtout impliquée par son corps et son affectivité. S'il peut exister des rites intimes, voire solitaires, où le lien social inhérent au rite sera d'essence spirituelle, il est le plus souvent vécu avec les autres; sa dimension communautaire l'emporte sur la dimension privée. Il est là pour s'adresser aux autres puisqu'il est dans sa nature d'affirmer des liens avec eux, si ce n'est même de les entretenir, voire de les créer.

Ces liens sont porteurs de significations symboliques qui s'expriment justement dans ces comportements plus que dans les paroles qui les accompagnent le plus souvent en renfort. Ces gestes, ces paroles, ces objets dont l'organisation séquentielle, rituelle, forme la trame du rite sont des symboles : derrière la matérialité de ce qu'ils sont, ils signifient autre chose qui est de l'ordre du culturel, du spirituel, de l'histoire originelle mythique ou religieuse du groupe qui s'y adonne. C'est bien en ce sens que le rite est codé. Il fait référence à des réalités non dites mais connues et exprimées indirectement, symboliquement. De ce fait, un rite a toujours un certain volume, une réelle épaisseur dans le temps et dans l'espace. Un rite ne se crée pas ; il s'instaure progressivement de lui-même en raison du consensus collectif qu'une bonne pratique reçoit peu à peu. Il finit par arriver à maturité puis, le plus souvent, se sclérose et disparaît. Tout ce processus demande du temps et doit nécessairement intéresser la majorité des constituants du groupe culturel ${ }^{27}$.

Définir les rites ou les rituels ${ }^{28}$ constitue une tâche ardue où le nouveau venu doit «éplucher» de nombreuses définitions pour parvenir à toucher tous les aspects essentiels à ce fait social que l'on cherche à

26. Denis Jeffrey, Éloge des rituels, Québec, Presses de l'Université Iaval, 2003, p. 13-14.

27. Michel Hanus, "Paroles, pratiques, rites et rituels », Ĺtudes sur la mort, n" 114, Rites et rituels, 1998, p. 14-15.

28 À l'instar de la très grande majorité des auteurs présentés dans cette recension des écrits, nous utilisons les concepts de rite et de rituel dans le mème sens. 
décrire, pour pouvoir ensuite l'utiliser comme concept et outil d'analyse. D'ailleurs, d'autres auteurs ont choisi la manière brève pour arriver à une définition du concept de rite, cherchant à en saisir l'essentiel, quitte à sacrifier de nombreux détails. Parmi eux, notons les suivants et leur énonciation :

- Jean-Yves Leloup et Marie de Hennezel : «Le rite est une tentative de maitriser ce qui échappe à notre compréhension en lui donnant du $\operatorname{sen} s^{29} \gg$.

- Victor Turner : l'action rituelle est « [...] une histoire qu'un groupe se raconte à lui-même ${ }^{30} »$.

- Denis Jeffrey : le rite est « [...] un acte symbolique qui donne à vivre du sens ${ }^{31}$ ». Dans un autre texte il dit : «Le rite soutient l'expression symbolique du refoulé ou de l'impossible à dire $[. . .]^{32} \gg$.

- Raymond Lemieux : “[...] le rite n'est rien d'autre qu'un aménagement symbolique fait de mots, de gestes et de mises en scène $^{33}{ }^{3}$.

- Isac Chiva : le rite est une " technique sociale symbolique ${ }^{34}$ ».

- Jean Maisonneuve : le rite "c'est un système codifié de pratiques, sous certaines conditions de lieu et de temps, ayant un sens vécu et une valeur symbolique pour ses acteurs et ses témoins, en impliquant la mise en jeu du corps et un certain rapport au sacré $^{35} \gg$.

- Henri Hubert et Marcel Mauss : les rites sont « des actes traditionnels d'une efficacité sui generis ${ }^{36}{ }^{36}$.

Jean-Yves Leloup et Marie de Hennezel, $L$ 'art de mourir. Traditions religieuses et spiritualité bumaniste face à la mort, Paris, Pocket, 1997, p. 180.

3" Victor Turner, "From ritual to theatre ", New York, Performing arts journal publications, 1982, p. 104. Cité par Claude Rivière, Les rites profanes... 1995, p. 32.

3 Denis Jeffrey, Filoge des rituels, op. cit., p. 219.

Denis Jeffrey, Jouissance du sacré, Paris, Armand Colin, 1998, p. 108.

Cité par Denis Jeffrey, ibid., p. 154.

it Cité par Jean Maisonneuve, Les conduites rituelles, Paris, PUF, [1988] 1999, p. 11.

Ibid., p. 12.

Cité par Jean Joncheray, "Rites et construction de l'identité : des rites de passage aux rites d'interaction et aux grands rites collectifs séculiers ", dans Bernard Kaempf (dir.), Rites et ritualités, Paris, Les Éditions du Cerf, Lumen Vitae et Novalis, 2000, p. 393. 
- Louis-Vincent Thomas : «Il y a rite, pour nous, chaque fois que la signification d'un acte réside dans sa valeur symbolique plus que dans sa finalité mécanique ${ }^{37} »$.

- Luce Des Aulniers : «Un rite, c'est un ensemble d'actes et de signes matériels à haute teneur symbolique, marquant à la fois l'expérience d'un changement perçu comme mystérieux, sinon menaçant pour l'existence - et appelant le dépassement ${ }^{38}$ ".

- Jean-Didier Urbain : «[...] une imagination organisatrice de la disparition... ${ }^{39} "$

Au-delà des efforts consacrés à la clarification du concept de rite, les études du dernier siècle ont été nombreuses à porter sur l'objet luimême et nous tenterons ici d'en retenir seulement les éléments utiles pour éclairer notre propos.

Les tentatives pour en arriver à une typologie des rites font penser aux essais de définition générale du rite. Plusieurs classifications sont nées sans qu'aucune ne permette encore de présenter, sans recoupements de catégories, les différents types de rites. Pour les besoins de cette étude, disons simplement, avec Pascal Lardellier ${ }^{40}$, qu'on s'entend pour distinguer les micro-rites, comme les rites d'interaction associés à la vie quotidienne (tels qu'analysés par Erving Goffman) et les grands rites communautaires (tels qu'analysés par Durkheim), dont les rites de passage sont probablement les plus connus et les plus étudiés. Nous nous référerons plus spécifiquement à ces grands rites communautaires puisque les rites funéraires appartiennent clairement à la catégorie des rites de passage.

La classification la plus célèbre reste encore celle d'Arnold Van Gennep $^{41}$ (1909) qui décrit les rites de passage (naissance, initiation, mariage, mort, entre autres) comme étant ceux où les participants sont

Jouis-Vincent Thomas, "La mort aujourd'hui : de l'esquive au discours convenu ", Religiologiques, n" 4, automne 1991, p. 12.

3* Luce Des Aulniers, Itinérances de la maladie grave. Le temps des nomades, Paris, Montréal, L'Harmattan, 1997, p. 554.

3) Jean-Didier Urbain, L'arcbipel des morts, op. cit., p. 23.

41 Pascal Lardellier, Théorie du lien rituel, op. cit, p. 21.

+1 Arnold Van Gennep, Les rites de passage, Paris, Picard, [1909] 1981. 
soumis à une séquence d'actes en trois temps ${ }^{42}$ : la séparation (d'avec le groupe social d'origine) ; la liminarité (mise en marge et séjour hors du monde social); la réintégration ou l'agrégation (entrée dans un nouveau statut).

Symboliquement, ces trois phases correspondent à une séquence mort/gestation/nouvelle naissance ${ }^{43}$. Même si elles existent dans tous les rites de passage, l'accent peut varier selon le type qui est célébré. Ainsi, pour les rites funéraires qui nous occupent plus spécifiquement, les rites de séparation sont plus développés (nous y reviendrons). Par cette médiation rituelle, le groupe veut insister sur une rupture et sur une transition d'un état social à un autre ${ }^{44}$, transition qui symboliquement ressemble à un passage physique, au franchissement d'un seuil dont on ne revient pas inchangé, et cette transition aménage et instaure un temps et un espace de coupure destinés à souligner la différence entre l'état antérieur et l'état postérieur.

Récemment, Jean Joncheray ${ }^{45}$ a posé un questionnement nouveau sur le thème des rites de passage qu'il convient de souligner ici. Il nous fait d'abord observer que Van Gennep, après avoir inventorié tous les types de rites de passage, fait remarquer que les plus typiques ont trait à l'initiation et particulièrement ceux qui sont associés au passage à la vie adulte. Pour lui, les rites auxquels on se prête à l'adolescence, dans les sociétés qui pratiquent l'initiation, sont une façon de faire comprendre, de mettre en scène, le fait que ce passage-là est comme un « concentré des autres passages », une prise de conscience que la naissance est déjà passage et que la mort sera aussi passage. Joncheray se demande alors si cette réalité ne tient pas au fait qu'au moment où Van Gennep publie ses travaux, la vie tout entière est justement vue comme un passage, $y$ compris la mort.

t2 L'anthropologue britannique Victor Turner proposera d'ajouter entre les étapes deux et trois une phase dite d'«action réorientéc ", c'est-à-dire d'engagement ferme des participants, en passe d'être initiés, dans une voie révélée par le rite et dont on accepte les droits et les devoirs.

4; Jean-François Dorticr (dir.), Le dictiomnaire des sciences bumaines, Auxerre, Sciences Humaines Éditions, 2004, p. 739.

t+ Notons que cela s'applique autant au défunt qu'aux endeuillés.

t5 Jean Joncheray, " Rites et construction de l'identité... ", op. cit., p. 393-414. 
Autrement dit, le rite du passage à l'âge adulte n'est possible que parce que l'ensemble de la vie est perçu comme un passage. Et à l'inverse, il est là pour rendre possible cette vision des choses. [...] Tout cela fonctionne parce que la vie tout entière est perçue comme passage, et pour qu'elle soit perçue ainsi ${ }^{46}$.

Joncheray fait l'hypothèse que l'analyse de Van Gennep, voulant que les rites funéraires soient des rites de passage, ne soit peut-être plus aussi valable aujourd'hui. En effet, si un nombre sensible de nos contemporains voient dans la vie quelque chose que l'on pourra bientôt rendre éternel ou encore comme devant mener directement au néant, toute la conception de la vie comme un passage est remise en cause. Et avec elle, peut-être, la logique des rites de passage, d'où, possiblement, les modifications importantes que subissent ces rites, dont les rites funéraires, au cours des dernières décennies.

\subsection{Pourquoi avoir inventé les rituels funéraires?}

À propos du rite dans les sociétés ancestrales, Cazeneuve s'exprime ainsi :

Pourquoi les primitifs s'interdisent-ils de manger certaines nourritures qu'ils ont à leur disposition? Pourquoi brúlent-ils les cabanes des morts? Pourquoi s'imposent-ils des mutilations, des privations? Pourquoi perdent-ils en des cérémonies un temps qu'ils pourraient occuper à améliorer leur existence? C'est en cela que réside l'irrationalité apparente du rite, d'autant plus que toutes les populations primitives ont de nombreux rites qui leur imposent beaucoup de contraintes. On est donc tout naturellement amené à se demander si, dans la condition humaine primitive, le rite n'est pas imposé par quelque nécessité cachée, ou si, du moins, il n'a pas une fonction qu'il faut tenter de découvrir par une analyse plus approfondie.

[...] La nécessité de la ritualisation, telle qu'elle apparaît dans la société primitive, est donc impliquée dans le fait que, par sa nature, l'homme ne peut ni s'enfermer dans sa condition, ni s'en échapper totalcment. L'homme est libre ou se croit tel, ce qui fait naitre en lui une certaine angoisse. Son action, son existence mème lui semblent comporter une marge d'indétermination, par là même d'insécurité. Il éprouve ainsi le sentiment de quelque chose d'autre, qu'il ne peut maîtriser. C'est ce que les anthropologues appellent, à la suite de Rudolf Otto, le numineux. Ce

40 Jean Joncheray, ibid, p. 398-399. 
terme, plus large que celui du sacré, désigne non seulement ce qui est surnaturel, mais qui se révèle comme mystérieux, à la fois attirant et effrayant. Ce qui échappe à la règle est inquiétant, et en même temps puissant. C'est pourquoi l'homme, quand il a le sentiment du numineux, de ce qui lui échappe, est tenté à la fois de s'en écarter et de s'en servir, ou bien, tout à la fois, de se préserver de ses dangers et de se mettre sous sa protection. De là résultent les attitudes fondamentales autour desquelles, en vertu de leurs fonctions anthropologiques, s'ordonnent les rites : la purification, la magie, la religion ${ }^{47}$.

En se référant à des auteurs comme Thomas, Des Aulniers, Baudry, Maisonneuve $^{48}$, Bacqué ${ }^{49}$, Lardellier ${ }^{50}$, Rivière ${ }^{51}$ et Jeffrey ${ }^{52}$, il est possible de tenter une synthèse faisant ressortir les principales fonctions des rites mortuaires.

De la façon la plus générale qui soit, les rites funéraires ont essentiellement trois finalités, selon Thomas ${ }^{5,3}$ :

1. Les rites s'adressent dans un premier temps au mourant et au mort lui-même. L'objectif visé est l'apaisement par le maternage au moment du mourir et la reconnaissance et le respect qu'on lui témoigne au moment de sa mort. Plus encore, le rite permet de "tuer le mort», selon l'expression des Mossi du Burkina Faso, parce qu'" il faut du temps pour mourir ", comme disent les Toradja d'Indonésie. En effet, la mort étant vue comme un passage, i] ne suffit pas de mourir pour que tout s'accomplisse. Il faut accepter de se séparer de notre proche décédé et, pour cela, on doit tuer ce qui reste vivant en lui en rompant les liens affectifs qui l'unissent à la communauté. Par la suite, après une période que chaque culture définit, il faut prévoir les rites permettant de réintégrer le mort confirmant ainsi le défunt dans son nouveau

47 Jean Cazeneuve, "Rite », dans Encyclopedia Universalis, Paris, 1985, p. 1156.

48 Jean Maisonneuve, Les conduites rituelles, op. cit., p. 13-14, 45-46.

49) Marie-Frédérique Bacqué (dir.), Mourir aujourd'bui. Les nouveaux rites funéraires, Paris, Odile Jacob, 1997, p. 85.

5.l Pascal Lardellier, Théorie du lien rituel, op. cit., p. 13-14.

51 Claude Rivière, Les rites profanes, op. cit., p. 27, 51-52.

52 Denis Jeffrey, Éloge des rituels, op. cit., p. 16-17, 39, 185, 198.

5.3 On retrouve ces trois fonctions du rite funéraire dans de nombreux textes produits par Louis-Vincent Thomas et aussi chez de très nombreux auteurs qui le citent. 
destin et conférant à ses restes leur statut définitif. Toujours selon les Mossi, le temps est alors venu de " faire revivre le mort ». Il s'agit donc, avec la première fonction du rite funéraire, de s'occuper tout à la fois du devenir corporel mais aussi spirituel du défunt. De plus, on le constate, la vie en société étant fondée sur les échanges entre individus occupant des places sociales distinctes, il ne suffit pas de mourir pour que le mort soit automatiquement repositionné ni pour que les vivants prennent automatiquement la place laissée vacante. C'est pourquoi non seulement les rites dits funéraires sont importants, mais également les rites de deuil, de commémoration et de levée du deuil, à la fois pour régler le sort du défunt, mais pour permettre également la réintégration des endeuillés dans le monde des vivants après une période plus ou moins longue de mise en marge. Il faut aussi noter - et c'est de toute première importance, même si on a beaucoup tendance à oublier ce service « matériel » que le rite rend aux sociétés - que les rites funéraires sont aussi là pour régler la question de la gestion du cadavre " que l'on a sur les bras ", question qui doit rapidement trouver une réponse pour éviter l'universelle horreur de la putréfaction.

2. Les rites funéraires, malgré le fait qu'ils semblent prendre appui sur la présence du cadavre ou de ce qui en tient lieu symboliquement, sont aussi centrés sur la prise en charge des survivants. Les rassurer, les apaiser, leur offrir un garde-fou contre l'angoisse existentielle, mais également permettre l'expression d'émotions intenses et déstabilisatrices pour l'individu et pour le groupe, voilà les finalités anthropologiques des rites à leur égard. La période de deuil est une importante zone de turbulences, une traversée du désert pour laquelle on ne veut pas abandonner celui ou celle qui s'y engage. En ce sens, encore une fois, les rites de deuil ${ }^{54}$ viennent compléter les rites funéraires pour faciliter et encadrer le travail de deuil et ainsi faciliter le rétablissement de l'ordre perturbé après expression codifiée de l'angoisse et après hommage au disparu.

54 Nous pensons, même si nous n'avons pas trouvé cette équivalence dans les écrits sur le sujet, que l'appellation "rites de deuil » devrait inclure celle de rites funéraires, puisque tout ce que l'on nomme rites funéraires constitue en fait des étapes visant à assurer la fonction thérapeutique universelle du travail de deuil. 
3. Finalement, les rites funéraires tiennent compte de la fragilisation du groupe social à l'occasion du décès de l'un de ses membres. Les forces en présence qui font irruption dans la communauté, la mort, le numineux, doivent être absolument tenues en respect par le recours aux rituels et à ses possibilités de mise en scène et de dramatisation contrôlée dans un cadre liturgique qui « font passer le drame du plan réel au plan de l'imaginaire ${ }^{55}$ ", c'est-à-dire là où l'humain retrouve un pouvoir sur les choses qui autrement le débordent et menacent à la fois la survie individuelle et celle du groupe. C'est là l'ultime pouvoir du rituel dans son affrontement symbolique avec une force, la mort, apparemment invincible : bien qu'elle réussisse à emporter l'un des nôtres et à priver la société de sa présence et de son apport, cette victoire apparente n'est que temporaire parce que, au bout du compte, à l'issue des rites funéraires et des rites de deuil, le mort ne sera plus un disparu ou un absent. Ce qui reste de lui sera « relocalisé » dans un ailleurs qui n'est pas totalement étranger au monde des vivants et la mort aura ainsi, encore une fois raté sa cible et la vie - celle du groupe essentiellement - pourra à nouveau être célébrée comme étant à l'abri des menaces d'anéantissement que faisait peser sur elle la mort. C'est ainsi que les rituels ont la fonction et la capacité de revivifier les solidarités d'une communauté.

En bout de ligne, à qui profite le rite? Selon Thomas :

Malgré leur disparité dans le temps et dans l'espace, les conduites funéraires obéissent à des constantes universelles. Leur finalité est double. En effet, au plan du discours manifeste, elles sont motivées par ce qu'elles apportent symboliquement au mort: par une série d'actions plus ou moins dramatiques, plus ou moins prolongées et parfois séparées par de longs intervalles, un lieu et des rôles lui sont assignés, en accord avec la continuité de la vie. Mais au plan du discours latent, même si le cadavre reste toujours le point d'appui des pratiques, le rituel ne prend en compte qu'un seul destinataire : l'homme vivant, individu ou communauté. Sa fonction fondamentale, inavouée peut-être, est de guérir et de prévenir, fonction qui revêt d'ailleurs de multiples visages : déculpabiliser, rassurer, réconforter, revitaliser. Socialement réglé, le rituel funéraire répond aux besoins de l'inconscient, prolongeant au plan de l'action, donc à travers les corps, les mécanismes de défense que l'imaginaire met en place pour

55 Louis-Vincent Thomas, Lescbairs de la mort, Paris, Institut d'édition Sanofi-Synthélabo, 2000 , p. 161. 
composer avec la peur de la mort. Ces mécanismes sont si subtils qu'il est souvent difficile de saisir les désirs authentiques par-delà les détours de la symbolisation ${ }^{56}$.

Thomas se résume en affirmant que le rite, avec son soutènement symbolique, existe en tant que thérapie universelle devant la menace et la peur de la mort. Luce Des Aulniers poursuit et précise sur le même thème.

Le rite autour de la mort s'élabore toujours en stéréophonie : à la fois pour le mort et pour les vivants. Ainsi, en célébrant le mort, la mort, on permet de médiatiser les forces qui menacent une collectivité. Comment? D'abord au plan psychosocial, parce que le rite, en dramatisant ponctuellement les sentiments individuels, évite leurs débordements ultérieurs, toujours menace pour l'ordre social ainsi que pour l'équilibre mental individuel : il a donc une fonction prophylactique. Ensuite, au plan psychoculturel, parce que le rite autour de la mort se saisit de cette occasion bouleversante pour inculquer des valeurs essentielles à une collectivité : il a donc une fonction éducative. De la sorte, les fonctions des rites dépasseront l'événement comme tel pour se projeter dans le temps social ${ }^{57}$.

Il faut insister ici sur l'universalité des rites funéraires. Comme le rappelle Patrick Baudry, « aucune société n'abandonne ses morts sans précaution rituelle ${ }^{58} »$.

Celui qui « part » oblige le groupe à traverser une crise que le rite a pour fonction de réguler. Une telle régulation [...] engage aussi le sens que cette communauté donne à l'existence devant le non-sens de la mort. Devant l'inconnaissable et l'impensable, la société ne fabrique pas seulement des significations apaisantes ou des représentations qui euphémisent le choc d'une mort dont on ne sait rien : c'est le soutènement symbolique de la vie sociale qui se trouve provoqué. La société redit devant la mort ce qu'elle est ou veut être ${ }^{5 !}$.

56 Jouis-Vincent Thomas, La mort, Paris, PUF, [1988] 2003, p. 92.

57. I.uce Des Aulniers, « Rites d'aujourd'hui et de toujours », dans Frontières, V ariations sur le rite, vol. 10, n" 2, hiver 1998, p. 4.

s\& Patrick Baudry, "La mise en scène de l'invisible », Frontières, I'ariations sur le rite, vol. $10, \mathrm{n}^{\circ} 2$, hiver 1998 , p. 7 .

54 Patrick Baudry, "La mise en scène de l'invisible ", op. cit., 1998, p. 7. 
Les rites funéraires assument également des fonctions communes aux autres types de rites en plus de celles qui leur sont propres $^{60}$ :

- maitriser le mouvant et les crises et réassurer contre l'angoisse en facilitant la transition lors des moments forts de la vie ;

- assurer la médiation avec le divin, l'occulte, le mystère, le numineux ou conjurer ces forces ;

- permettre la mise en scène du langage symbolique donnant ainsi accès à l'imaginaire qui vient à la rencontre ou à la rescousse d'une réalité trop crue ;

- permettre la communication et l'échange ou les réactiver ;

- garantir la régulation sociale dans une optique d'ordre et de cohésion à maintenir ou à retrouver, tout en légitimant cet ordre ;

- domestiquer ou canaliser la violence des pulsions (et, dans certains cas, au contraire, susciter une forme de catharsis) ;

- assurer, par la socialisation, la transmission et l'appropriation d'une mémoire collective qui fournit à l'individu une matrice de réponses déjà inscrites dans un ordre social et lui permettent de s'intégrer à une culture et de confirmer son appartenance identitaire ;

- assigner des rôles et différencier des acteurs sociaux tout en garantissant leur collaboration dans le contexte rituel.

Les rites ont pour fonction, dans une certaine mesure, de montrer ce qui est caché. «La ritualité nous force à prendre connaissance de ce que nous savons sans y croire ${ }^{61} \gg$. Pour ce faire, les rites mettent en actes une symbolique capable de donner un sens aux limites de la condition humaine en simplifiant la pensée par des gestes universels et, en connectant chacun, par le biais des échanges, avec une humanité commune, en solennisant les temps forts de la vie tout en canalisant et en pacifiant des émotions puissantes.

(11) Il est à noter toutefois que le fait de tenter d'inventorier ces fonctions ne signifie pas qu'elles soient toujours présentes ou jouent toutes de la même façon dans tous les types de rites.

"1 Patrick Baudry cité par Marie-Frédérique Bacqué, "Introduction. Retrouver l'émotion ", dans Marie-Frédérique Bacqué (dir.), Mourir aujourd'bui. Les nouveaux rites funéraires..., 1997, p. 13. 


\subsection{Les rites comme espace et système de communication ${ }^{62}$}

Pour jouer correctement certains types de musique, il faut pouvoir compter sur un orchestre assez élaboré. Par analogie, pour communiquer certaines réalités, il faut le rite. La musique bien rendue qui parvient à notre système auditif est une réalité riche, complexe, multidimensionnelle et encodée. Ce qui parvient à nos sens dans des situations de communication en contexte rituel possède des caractéristiques identiques. Par contre, affirmer que le rite est essentiellement communicationnel ne revient pas à dire qu'il est un système de communication comme les autres.

Une première façon de distinguer divers systèmes de communication pourrait compter deux critères : le nombre de participants engagés et le degré de formalisme présent. De là, on peut tirer quatre contextes croisés de communication (communication interpersonnelle ou alors sociale, formelle ou alors informelle). Les rituels étudiés ici, c'est-à-dire les rites funéraires en tant que rites de passage, s'inscrivent clairement dans un contexte de communication sociale de type formel.

Ce type particulier de communication compte sur un contexte et un dispositif rituels permettant une communication à deux niveaux. D'abord, entre les participants à la cérémonie rituelle, puis - et là réside l'une des caractéristiques essentielles du rite - entre l'ensemble de ces participants et des entités ou des représentations mythiques. On dépasse ici le modèle classique de la communication avec émetteur et récepteur par la voie d'un code linguistique. Le rite devient clairement outil de médiation par lequel transitent des formes de métamessages auxquels on a accès via une "performance " utilisant surtout des codes extralinguistiques faisant largement appel aux actes accomplis par des corps plus encore que par le recours au discours ${ }^{63}$. Le résultat potentiel

6.2 Cette section du texte doit beaucoup à l'ouvrage de Pascal Lardellier (Théorie $d u$ lien rituel. Anthropologie et Communication, Paris, L'Harmattan, 2003) qui tente de faire le pont entre les savoirs de l'anthropologie et des sciences de la communication sur les rituels, et plus particulièrement à son. chapitre 3 intitulé « Rite, médiation, communication », p. 99-129.

6.3 Roy A. Rappaport (Ritual and religion in the making of bumanity, op. cit., p. 139-144) souligne à ce sujet que bien que les humains possèdent la capacité de parler, leurs rituels comprennent pourtant des actions autant que des paroles, et, dans plusieurs 
actualisé par le contexte et le dispositif rituels, c'est d'instituer une situation de communication dans une relation plus organique, une relation d'union ou même de communion entre deux groupes auparavant dissociés : le(s) célébrant(s) et les fidèles, pour conserver les vocables ecclésiastiques. Ajoutons que, à cette occasion, deux types de messages peuvent être transmis par les participants : les « self-referential messages » et les autres baptisés «canonical messages » par Rappaport ${ }^{64}$. Les premiers, également utilisés par les animaux dans divers contextes, permettent aux acteurs d'informer les autres participants sur leur propre état physique, social ou psychique (attitudes d'agression, de soumission ou de fuite par exemple). Les seconds, inconnus du reste du règne animal, concernent des messages additionnels transmis par les mêmes acteurs bien qu'ils ne soient pas encodés par eux. Ils les trouvent déjà encodés dans la liturgie (par exemple, certains mots ou gestes utilisés dans le rituel liturgique d'une cérémonie catholique depuis plus de 1000 ans). Le premier type de message représente l'immédiat, le particulier et les aspects vitaux de l'événement ; le second représente, par contraste, le général, le durable ou même les aspects éternels, invariants, de l'ordre universel. Les deux types de messages ne sont pas transmis dans des rituels distincts, mais sont entremêlés dans les mêmes rituels.

Par ailleurs, le pouvoir de médiation joué par le rituel est double. Il agit tout autant sur le plan technique, par le biais du dispositif rituel (lieu, décor et décorum), que sur le plan symbolique, grâce au contexte rituel (performance « jouée » et manipulation de symboles qui sont autant de signes prenant la place de choses ou d'entités absentesprésentes, invisibles-visibles, indicibles-évoquées). En bout de ligne, le

cas aussi des objets et des substances qui sont manipulés pendant le rituel. Il pourrait s'agir, à première vue, d'un archaïsme remontant à une époque où l'humain ne disposait pas du langage pour communiquer. Mais pourquoi alors cette façon de faire se serait-elle maintenue jusqu'à aujourd'hui ? Pourquoi les humains qui peuvent communiquer avec aisance, efficacité et subtilité par le biais du langage emploient-ils encore le mode de communication plus physique du corps? Il parait évident que c'est parce que le fait de montrer, d'exhiber, de parader le corps (consciemment ou non) indique davantage, plus clairement ou autrement que les mots, ce que l'on souhaite communiquer dans certaines situations. L'acte vient ajouter du poids aux paroles. II est souvent considéré comme une démonstration qui va plus loin. Si la parole est une forme d'action, laction est aussi certainement une manière de dire ou de parler.

ot Roy A. Rappaport, ibid., p. 52-54. 
rituel tire son efficacité, qu'on peut qualifier d'extra-empirique, de cette double médiation technique et symbolique. Un recours intensif aux symboles est donc caractéristique du déroulement du rite. Le symbole est, pour reprendre les mots de Luce Des Aulniers, «cet apparent petit rien qui évoque tant; ce quelque chose dont on sait si peu, bien gardé par l'inconscient, lancé vers un univers dont on ne sait rien ${ }^{6.5}$ m. Edgar Morin ajoute qu'il faut savoir que le symbole a beau être une représentation d'autre chose, il porte néanmoins en lui toute la puissance émotive, toute la chaleur ou, au contraire, toute l'horreur de ce qu'il évoque ${ }^{66}$.

Il faut aussi insister sur le fait que le rite engendre ou permet deux types de communication. Au premier plan, il permet la communication sociale, au sens large, entre un grand nombre de participants. Plus important encore, il favorise, à un autre niveau, une intensification des interactions sociales et des contacts lors de la préparation du rite, de son déroulement et même dans l'après-rite. Cet espace et ce temps où se joue la « liminarité rituelle » permettent aux participants d'entrer dans une « anti-structure sociale ${ }^{67}$ ", c'est-à-dire de créer des liens sociaux temporaires - hors hiérarchie - mais potentiellement très intenses, d'où le sentiment de communion qui peut en découler. Ce contexte rare et très particulier permet un brassage social et culturel riche et élargi et c'est pourquoi Lardellier utilise la notion de «creuset rituel » qui non seulement permet des formes de communication sociale extralinguistique, mais agit comme un accélérateur des relations sociales entre des gens réunis pour la même chose. Voilà d'ailleurs un troublant paradoxe du rite comme système de communication, marqué à la fois par des relations sociales très formelles, mais également très intenses.

Par la médiation rituelle, les institutions et les groupes existent par la communication mais aussi dans la communication, se situant à la fois dans le temps et en dehors du temps (le temps rituel est un temps désenclavé, sorti du temps de la quotidienneté), dans toute structure sociale et au-dessus d'elle.

6.5 Luce Des Aulnicrs, « Rites d'aujourd'hui et de toujours »..., op. cit., p. 6.

66 Edgar Morin, L_bomme et la mort, op. cit., 1970, p. 106.

6.) Victor Turner, Le phénomène rituel. Structure et contre-structure, Paris, PUF, 1990, cité par Pascal Lardellier, Théorie du lien rituel, op. cit., 2003, p. 108. 
La symbolique des rites funéraires constitue un langage qui exerce une action particulièrement complexe qui procède, selon Louis-Vincent Thomas, tout à la fois par suggestion/occultation, dramatisation/dédramatisation et intégration/exclusion.

Le symbole suggère probablement plus qu'il n'explique. Sa fonction joue davantage peut-ĉtre au niveau moteur et affectif qu'à celui de l'intelligence proprement dite. D'où l'importance du sensible comme «révélateur».

Mais le symbole ne soulève qu'imparfaitement le masque. Il « cache en dévoilant et révèle en cachant ». À bien des égards, on peut admettre qu'il occulte - ce qui est vrai surtout pour celui qui ne le « lit » pas (l'étranger, l'autre, le non-initié), mais l'est aussi partiellement pour celui qui s'en réclame.

De fait, il dit beaucoup mais pas tout; c'est une puissance qui tente de surmonter une grande impuissance, ce qui faisait déclarer à George Gurvitch que le symbole apparait chaque fois qu'il «faut manier des obstacles ».

Si en un sens nous pouvons dire du symbole qu'il révèle, il est tout aussi juste de dire qu'il «mystérise ». C'est bien ce que met en évidence sa visée hiérophanique ; il en dit suffisamment pour établir un contact avec le numineux, pour favoriser un échange de vie et d'énergies vitales entre l'orant et les puissances, mais pas assez pour que la connaissance soit parfaite, totale, immédiate (au sens fort de "sans médiation ») et à plus forte raison définitive; il donne l'envie d'aller plus loin afin de saisir l'invisible, l'inaudible, l'indicible ${ }^{6 \beta}$.

\subsection{Mort, rites et création sociale}

Dans L'homme et la mort, Edgar Morin rappelle que « [...] l'espèce humaine est la seule pour qui la mort est présente au cours de la vie, la seule qui accompagne la mort d'un rituel funéraire, la seule qui croit en la survie ou la renaissance des morts ${ }^{(0)}$ ». Pour lui, l'invention de la sépulture est tout aussi déterminante pour notre humanité que ne l'a été la fabrication des premiers outils : l'outil et la sépulture sont tous deux des "phénomènes humains premiers ", la marque du passage de la nature à la culture. Pour lui, nous sommes dès lors devant un triple donné anthropologique ${ }^{71}$. D'abord, notre ancêtre a « conscience de

68 Jouis-Vincent Thomas, Les chairs de la mort, op. cit., p. 203-204.

(6) Edgar Morin, L'bomme et la mort, op. cit., p. 17 de la préface à la deuxième édition.

(1) Ibid., p. 42-43. 
l'événement de la mort », de la rupture qu'elle représente et, surtout, il l'associe à la perte de l'individualité, celle de l'autre, mais aussi, par extension, la sienne propre. Cette nouvelle donne provoque des émotions violentes que Morin qualifie de «traumatisme de la mort». En conséquence, l'humain développe et affirme vigoureusement sa "croyance en l'immortalité », autrement dit le maintien de son individualité après la mort. Dès l'aube de la pensée humaine, la sépulture exprime donc une sorte de révolte active contre la mort. C'est sur cette base qu'il peut poursuivre son analyse en parlant du rôle central de la mort dans la création de la vie sociale.

[...] la société fonctionne non seulement malgré la mort et contre la mort (en sécrétant notamment une formidable néguentropie imaginaire où la mort est niée et refoulće), mais [...] elle n'existe en tant qu'organisation que par, avec et dans la mort. L'existence de la culture, c'est-à-dire d'un patrimoine collectif de savoirs, savoir-faire, normes, règles organisationnelles, etc., n'a de sens que parce que les anciennes générations meurent et qu'il faut sans cesse la transmettre aux nouvelles générations. Elle n'a de sens que comme reproduction, et ce terme de reproduction prend son plein sens en fonction de la mort ${ }^{\prime \prime}$.

Plus près de nous, en 2004, Jean-Claude Métraux parle à son tour du potentiel créateur de la mort par l'intermédiaire du deuil qu'elle fait vivre à chacun. «Toute tradition mortuaire permet en effet de perpétuer ou restaurer l'identité narrative d'une communauté écorchée par la mort de ses membres ${ }^{72}$ ». Pour cet auteur, la mort et le deuil ont un rôle central dans toute création humaine, en particulier lorsqu'il s'agit de création de sens.

Par ailleurs, en évoquant le potentiel de création ou de recréation sociale de la mort, Anati fait remarquer que les rites funéraires contribuent toujours à la constitution et à la reproduction du groupe social en tenant compte d'un système de places et de rôles.

Dans les rites d'initiation ou dans les rites funèbres, dans les rites préparatoires à la chasse ou dans l'évocation des exploits des ancêtres, chaque membre du clan a son rôle, qui dépend du sexe, de l'âge, du rang ou de tout autre facteur d'identification. $[. ..] \dot{A}$ travers le rite, chacun réaffirme son rôle dans la société et confirme son identité propre. Ce

\footnotetext{
"I Ibid, p. 12-13.
}

72 Jean-Claude Métraux, Deuils collectiffs et création sociale, Paris, La dispute, 2004, p. 208. 
facteur structurel est présent chez les peuples tribaux des cinq continents ${ }^{73}$.

Et Éric Volant de renchérir :

Le décès d'un proche est signe de rupture. Un ordre acquis du monde s'en trouve déchiré et une place est laissée vide. L'enjeu majeur du rituel funćrairc, c'est sa capacité de restructurer le tissu social et d'inscrire les subjectivités individuelles dans un réseau de places et de rapports sociaux reconstitués autrement ${ }^{74}$.

Si la mort et les rites concourent à la création des sociétés humaines dans un sens large, examinons ici maintenant les rites funéraires en tant que contexte social particulier permettant la mise en place de rapports sociaux d'un type différent. Chaque fois qu'un groupe doit affronter la perte de l'un de ses membres, il se déclenche une série de réactions et d'actions pour mettre en place et en scène les rites de mort. Survivants et vivants se retrouvent autour des trois fonctions ou finalités universelles des rites funéraires telles que nous les avons exposées préalablement. Des rapports sociaux s'instituent donc dans l'avant-rite ou dans la préparation du rite. Il faut que quelqu'un prenne en charge la préparation du corps du défunt pour que le rite puisse avoir lieu le moment venu. On s'occupera aussi d'accompagner les proches endeuillés et de les entourer avant et pendant le rite et normalement aussi après, pour ce qu'il est convenu de nommer la période de deuil ${ }^{75}$. Toute la communauté est donc occupée et ne peut que tenir compte de cette nouvelle provocation de la mort.

Pendant les rites funéraires, nous assistons aussi de façon claire à des rapports sociaux distincts de ceux auxquels nous participons habituellement. Rappelons que les rites de passage, rites communautaires par excellence, supposent la participation d'un grand nombre de personnes dans un cadre formel et où la communication sociale prend des formes plus symboliques que discursives et où les divers acteurs ont à tenir des rôles précis et distincts de ceux qu'ils tiennent en d'autres

Eimmanuel Anati, La religion des origines, op. cit., p. 14.

-4 Éric Volant, "La religion et la mort », op. cit., p. 333.

-5 Cette période de deuil est très variable dans sa forme et dans sa durée selon les groupes culturels et les périodes étudiées. De même, les obligations des membres de la communauté à l'égard des endeuillés sont également très variables. Finalement, on a pu observer dans les sociétés occidentales modernes une tendance à la privatisation du deuil. Nous aurons l'occasion de revenir sur cette question. 
temps. Le résultat attendu est une forme de communion sociale faisant que des solidarités soient réaffirmées. Les rites sont également l'occasion d'un apprentissage social et ils permettent de socialiser les membres d'une collectivité à un héritage commun remontant à un mythe fondateur. En d'autres mots, les rites donnent lieu à la création de rapports sociaux riches et distincts de ceux que la vie sociale habituelle permet dans le cadre des autres activités sociales.

Les rites mortuaires, et cet aspect est plus inattendu, permettent aussi la création de rapports sociaux entre les morts eux-mêmes. En effet, l'un des objectifs des rites funèbres est de régler bien sûr le sort du cadavre, mais surtout le devenir eschatologique de ce qui lui survit, que ce soit l'âme, l'esprit ou le double selon les divers systèmes de croyances. Ce faisant, le rite permet au défunt de joindre le groupe des morts et de l'intégrer à un lieu et à des fonctions nouvelles: monde des ancêtres pour les uns, "communion des saints " pour les autres, entrée dans un nouveau cycle de réincarnation, attente dans un entre-monde, etc. Bien au-delà d'une assignation topographique du cadavre, le rite règle donc aussi une assignation eschatologique pour la part qui lui survit.

Ce faisant, le rite instaure aussi des rapports sociaux nouveaux entre les vivants et les morts. On l'a vu, le rite doit à la fois reconnaitre la réalité et l'inéluctabilité de la mort de celui qui nous quitte (et en ce sens il nous en sépare) et lui assigner un nouveau " lieu de résidence », mais il doit aussi s'assurer que la frontière entre le monde des vivants et celui des morts n'est pas complètement étanche (et en ce sens il permet une certaine communication, que l'on définit différemment selon le système de croyances adopté). Les rites funéraires créent donc des rapports sociaux entre les vivants et les morts non seulement au moment des cérémonies elles-mêmes, mais bien au-delà. D'ailleurs, comme l'a rappelé Auguste Comte, les sociétés humaines comptent bien plus de morts que de vivants dans leur histoire ${ }^{76}$, et les vivants d'aujourd'hui, même s'ils n'en sont pas toujours conscients, vivent dans un environnement

Dans son roman L'immortalité, Milan Kundera avance le chiffre de 80 milliards et pendant les deux derniers siècles seulement, près de 9 milliards d'hommes ont cessé de vivre comme le rapporte Louis-Vincent Thomas dans Mort et pouvoir, Paris, Payot, 1978. 
où la "puissance des morts" ${ }^{77}$ ", pour reprendre une expression de Peter Berger, est tangible à travers tout ce dont on a hérité des générations antérieures.

J.'homme ne prend d'ailleurs pas seulement soin de ses morts dans le sens où il se plait à souligner pour lui-même et pour les générations à venir les traces de leur passage parmi les vivants et à se donner les preuves de leur inscription dans la mémoire collective : il prolonge leur geste existentielle, il accomplit leur volonté, il puise sa légitimité dans cette allégeance et cette filiation qui font de lui un descendant et un héritier. En ce sens, on a pu dire que le propre de l'homme était encore d'être de part en part "nécrocratique ": "En tant qu'Homo sapiens, nous naissons de nos parents biologiques. En tant qu'êtres humains, nous naissons des morts - du terroir qu'ils occupent, des langages qu'ils ont habités, des mondes qu'ils ont construits, des nombreux héritages institutionnels, légaux, culturels et psychologiques qui, à travers nous, les relient à ceux qui sont encore à naitre ${ }^{-k}$. Pour toujours l'homme qui vient au monde est un porte-parole, le porte-flamme d'une humanité qui s'est tue, éteinte, et dont il assure la survie ${ }^{-y}$.

Jean-Marie Brohm résume bien ces rapports sociaux complexes créés à répétition à l'occasion de la mort d'un individu.

De manière plus générale c'est donc la fabrication de la mort et des morts qui permet de construire la réalité sociale, laquelle articule indissolublement les liens entre les morts, qui continuent de survivre dans le souvenir des vivants ou dans "des lieux de vie " et "lieux de mémoire "spécifiques (morts vivants en tant que défunts), entre les morts et les vivants, lesquels sont des morts en sursis ou des survivants provisoires, et entre les vivants qui sont héritiers, représentants de morts, mais aussi potentiellement des «tueurs" ou des fabricants de mort. De même que les vivants sont territorialisés par leurs morts (la terre des ancêtres), les morts sont assignés à résidence par les vivants dans des espaces-temps circonscrits qui sont des « lieux de mort » : lieux de conservation (cimetières, catacombes, caveaux, ossuaires, nécropoles), lieux mythologiques (les différents royaumes des trépassés, des âmes mortes, des ancètres), "lieux psychiques " où ils continuent malgré tout à exister pour nous (urnes funéraires, lettres, photos, films,

Peter Berger, Comprendre la sociologie, Paris, Éditions du Centurion, 1973, p. 123.

7* Robert Harrisson, les morts, Paris, Le Pommier, 2003.

" Frédéric I.enoir et Jean-Philippe de Tonnac, "Introduction », dans Frédéric Lenoir et Jean-Philippe de Tonnac (dir.), La nort et l'immortalité. Encyclopédie des savoirs et des croyances, Paris, Bayard, 2004, p. 26. 


\section{LUC BUSSIÈRES / RITES FUNËBRES ET SCIENCESHUMAINES...}

enregistrements sonores, effigies), lieux surnaturels où ils survivent en tant qu'êtres de l'au-delà (spiritisme, etc. ${ }^{80}$.

2.5. Des idéologies funéraires pour affronter le face à face avec la mort.

La façon de concevoir la vie et la mort ne se règle pas au hasard. En tant qu'élément important et central dans l'organisation sociale, elle relève du monde des idéologies, donc des visions du monde qui fondent l'organisation du social. Les réflexions humaines concernant le destin de l'humain après la vie, que l'on nomme eschatologies, nous présentent un éventail somme toute assez restreint de systèmes de croyances ou d'idéologies à propos de la mort.

Philippe Gaudin ${ }^{81}$ en fait une analyse assez détaillée où il distingue, par exemple, les eschatologies selon les formes de vie qui sont réservées aux défunts. La mort peut, selon le cas, correspondre à une forme de vie diminuée, morne, où les morts sont réduits à des ombres sans force, sans mémoire ; à une vie exaltée, au sens positif ou négatif, par rapport à ce qu'est la vie terrestre ; à une existence terrestre invisible dans le village des morts; à une forme de vie dissociée où seule une part de l'humain lui survit; à un anéantissement de la personne jusqu'à ce qu'une intervention divine la recrée. Gaudin avance que l'on peut aussi classifier les eschatologies selon la vision du temps qu'elles mettent de l'avant, soit linéaire, soit cyclique.

Pour Edgar Morin ${ }^{82}$, il est possible de déceler seulement deux visions eschatologiques premières, "ethnologiquement universelles», lesquelles expliquent ensuite la formation des autres par syncrétisme. Dans les consciences archaïques, la première croyance qui serait apparue serait celle de la "mort-renaissance " par transmigration. Dans ce cas, tout mort humain renait, tôt ou tard, sous la forme d'un enfant ou d'un animal. Le second type à voir le jour est la «mort-survie du double ». Avec cette croyance, apparaît la volonté de sauver notre intégrité, notre

81) Jean-Maric Brohm, dans sa préface au livre posthume de Louis-Vincent Thomas, Les chairs de la mort, op. cit., p. 30-31.

${ }^{81}$ Philippe Gaudin, La mort. Ce qu'en disent les religions, Ivty-sur-Seine, Les éditions de l'A telier, 2001, p. 20.

82 Edgar Morin, L'bomme et la mort, op. cit., p. 123-172. 
individualité, par-delà la décomposition. C'est l'univers du « double ». Les morts ne sont ni désincarnés ni très loin des vivants. S'ils sont invisibles, ils n'en ont pas moins un corps, à la manière d'un spectre ou d'un fantôme, à l'image exacte du vivant qu'ils ont été, sans en être la simple reproduction post-mortem. En effet, le double accompagne le vivant dans toute son existence, disposant d'une force surnaturelle, et il veille et agit pendant que le vivant dort et rêve. Le vivant est d'ailleurs conscient de l'existence de son double; il le voit et le sent, entre autres moyens, par son ombre, par son reflet et par son souffle.

Louis-Vincent Thomas présente, quant à lui, quatre types eschatologiques possibles ${ }^{8.3}$ :

- la néantisation totale : qui est, selon lui, rare même chez nos contemporains chez qui il y aurait toujours persistance d'une quelconque espérance d'un prolongement de soi ;

- la vie indéfinie : croyance qui s'appuie sur la foi en les progrès de la science biomédicale qui arrivera un jour à stopper le vieillissement et à supprimer la mort considérée comme une maladie ;

- les traces : d'autres se contentent de survivre dans leurs enfants porteurs génétiquement d'une partie d'eux-mêmes, dans leurs disciples, dans leurs oeuvres matérielles, sociales ou intellectuelles;

- la renaissance ou la métamorphose : purgatoire, enfer, ciel et résurrection n'entraînent plus autant l'adhésion aujourd'hui, selon lui. Par contre, la réincarnation connaît un regain de popularité, s'appuyant sur l'idée qu'une vie n'est pas une expérience suffisante pour définir la destinée éternelle.

En fait, on peut aussi, avec DeSpleder et Strickland ${ }^{84}$, choisir de ramener à deux perspectives seulement nos diverses entreprises eschatologiques dans l'histoire : ou bien la mort mène à une porte, à un seuil ou à un passage - pouvant prendre des formes très diverses - ou, à l'opposé, elle aboutit à un mur, au néant. Georges Balandier exprime lui aussi la même idée.

D'un còté la mort, l'inéluctable, qui fixe à jamais dans la disparition, d'un autre côté l'espérance, la projection dans un devenir tout autre où la mort et le destin ont perdu leur emprise. L'une soumet par l'achèvement, l'autre

8.3 Louis-Vincent Thomas, La mort, op. cit., p. 113-117.

${ }^{4} 7$ Lynne Ann DeSpelder et Albert Lee Strickland, The Last Dance, Mountain View, Mayfield Publishing Company, 1996, p. 586. 
libère par le dépassement ou la transfiguration. I.'une a le temps pour allié, l'autre le réduit à l'effacement dans l'éternité ou à l'enfermement sans terme, par la succession des cycles d'une vie humaine continuéc dans ses transformations sans fin. L'une fonde sa victoire sur la précarité humaine, l'autre s'y oppose en conduisant l'avancée vers plus de perfection, vers moins de vulnérabilité. L'espérance moderne suprême cst celle qui se forme avec l'attente du moment où la mort pourra être "tuce "; la figure de l'" amortalité " se constitue alors dans l'imaginaire ${ }^{85}$.

Ce bref bilan illustre le fait que l'humanité a vu évoluer des idéologies où la survie de notre âme était assurée, où l'existence de cette âme et de la survie étaient sérieusement mises en doute, et où finalement, la mort elle-même était niée. «L'époque que nous vivons en Occident se trouve, quant à l'eschatologie, en pleine mutation; elle soulève aussi plus d'une ambiguitét ${ }^{86} »$.

Ces idéologies funéraires auront, il va sans dire, des impacts sociaux assez différents dans les sociétés où elles sont véhiculées. Leur association avec les rites funéraires mis au point par nos ancêtres est significative. Ces rites possèdent d'ailleurs un caractère d'invariant anthropologique en ce que, partout, ils ont constitué un déni symbolique de la mort, en limitant le pouvoir dissolvant de la mort à notre corps, laissant ainsi intacte une autre partie de la personne pour une forme de survie après ce passage. Pour la majorité des auteurs consultés, cette forme de déni est salutaire pour l'équilibre individuel et social. À l'instar de Louis-Vincent'Thomas, Patrick Baudry affirme que : «Contrairement à une idée reçue, la ritualité ne permet pas d'« apprivoiser » la mort. À l'inverse elle met en scène le refus culturel de la mort, ou disons plutôt le refus, essentiel à la culture, d'intégrer la mort ${ }^{87} 》$. En effet, pour Baudry, la mort demeure un arrachement, une rupture, une menace, même quand certains discours affirment qu'elle fait partie de la vie. Voilà une importante ambivalence puisqu'elle en constitue tout à la fois son contraire. On ne peut donc prendre la mort à la légère, malgré le travail des rites.

Essentiellement, la ritualité funéraire convoque le groupe devant la limite de la mort, et ce faisant oblige au rappel des interdits majeurs qui fondent

\footnotetext{
".5 Georges Balandier, «D'une espérance à l'autre. L'émergence de l'homme amortel 》, dans Frédéric Lenoir et Jean-Philippe de Tonnac (dir.), op. cit, p. 871.

so I.ouis-Vincent Thomas, La mort, op. cit., p. 113.

87 Patrick Baudry, "La mise en scène de l'invisible », op. cit., p. 7.
} 
l'humanité. Parce qu'on ritualise la mort, on ne l'accepte pas davantage. [...] Ce que l'on accepte, ce que l'on doit accepter, ce n'est pas « la mort», dont on ne sait rien, mais ce rapport [à la mort] culturellement construit. I.obligation majeure de la ritualité funéraire consiste à devoir se positionner devant la réalité du décès dans la condition sous laquelle le rapport à la mort se travaille culturellement et rend possible cette position $^{88}$.

Ainsi, non seulement l'individu, mais le corps social peuvent se permettre d'exister sans craindre à tout moment l'irruption de la mort dont les effets restent graves mais pas incontrôlables. Comme l'explique Jean-Didier Urbain, on fait face à la mort de multiples façons. Il s'agit d'un « [...] acte essentiel de représentation qui estompe et la violence absolue de la rupture et l'énigme de son au-delà. [...] La mort de l'autre ou de soi perd soudain ce coefficient de sauvagerie et de mystère qui la rend insupportable $e^{89}$. Ainsi, l'intelligence humaine, au sens collectif, «ne nie pas la mort mais l'organise - en refusant de la concevoir comme un arrachement définitif, irréversible séparation ou néantisation ${ }^{90} »$.

Par contre, avec le développement des visions eschatologiques portant sur la néantisation et l'amortalité, ce qui change maintenant, c'est que le déni de la mort est réel, non plus symbolique, en ce sens que c'est la mort elle-même qui est niée dans la réalité. En effet, on veut bien accepter l'idée que la mort est la terminaison de la vie individuelle, qu'elle intervient au bout de la vie. Ce faisant, on la situe ainsi hors de l'existence et elle ne vient plus en apparence déranger la vie. Or, c'est le contraire qui est vrai : la mort est présente dans la vie individuelle et collective et elle les détermine de multiples manières, entre autres, en obligeant la société et ses membres à se situer devant la limite qui les fonde. La logique et l'attitude contemporaines d'« acceptation » de la mort comme incident final sont résolument piégées comme le montrent bien Patrick Baudry et Henri-Pierre Jeudy.

Ce n'est pas un groupe qui se sépare du mort, mais le défunt qui, par son décès même, s'en est définitivement absenté. La cessation des fonctions vitales constitue la preuve qu'il n'est plus là. Et donc qu'il n'y a plus à «rester là ». Le mort n'est pas cette personne qu'il faut conduire jusqu'à sa dernière demeure, mais l'individu disparu, celui qui s'est définitivement

H8 Ibid., p. 8.

*. Jean-Didier Urbain, $L$ 'archipel des morts, op. cit., p. 26. En italique dans le texte.

Ibid., p. 26. 
absenté de lui-même, c'est-à-dire de son habitacle corporel. [...] Ici, l'individu décédé est celui qui se désabonne de lui-même. Inutile donc de mettre en scène une séparation, de ritualiser la distanciation entre morts et vivants ou d'assurer, dans la prise même de cette distance, la permanence du souvenir, en retenant l'autre en un lieu. Le décès tient du débranchement final. [...]

Ce qui est remarquable, c'est que la violence de la mort puisse se régler tout aussi violemment, mais sous couleur d'une résolution rationaliste de la cessation de l'existence et d'une gestion pacifiée du cadavre. La pacification est un processus qui empêche l'expression de la violence : elle est une forme de "sur-violence"; la violence consistant, notamment ici, dans l'interdiction de dire et ritualiser la brutalité d'un événement, ou dans la réduction d'une perturbation à une "information $"$ ".

En convertissant la mort en disparition, on escamote la présence et la force agissante de la mort dans la vie et on prétend avoir affaire à un incident individuel alors qu'une élaboration culturelle de cette mort a toujours été retenue comme nécessaire jusqu'à maintenant. Le travail du rite est de faire reconnaitre aux vivants un fait brutal, la mort. Ce qui vient ensuite, le travail de pacification ou «d'acceptation » de ce fait, ne peut faire l'économie de cette souffrance, de ce choc initial et brutal.

En résumé, pour des auteurs comme Thomas et Baudry, le déni symbolique de la mort est salutaire en ce qu'il permet d'intégrer la mort à la vie dans la culture; c'est le déni réel de la mort comme faisant partie de la vie - comme ayant le pouvoir de faire irruption dans la vie - qui pose problème à leurs yeux, individuellement et socialement. Le premier existe depuis que les rites funéraires sont nés; le second apparaît vraiment avec la modernité et avec notre nouveau rapport à la mort et il change du même coup, c'est évident, notre rapport aux rites et à la vie.

\subsection{Des rites pour gérer la disparition}

Si les rites ne sont pas indépendants des idéologies eschatologiques qui traversent une société ou une histoire, ces rites sont en même temps plus que des systèmes d'idées ou de croyances et plus que des systèmes de communication. Ils constituent aussi d'efficaces systèmes d'action et de gestion pour régler la priorité de tout rite funéraire, c'est-à-dire le

") Patrick Baudry et Henri-Pierre Jeudy, Le deuil impossible, Paris, Éditions Eshel, 2001, p. 47. 
“ comment se débarrasser du cadavre" " et la manière à adopter pour couper les vivants des transformations biologiques associées à la putréfaction qui ne manqueront pas de se manifester très tôt.

En effet, la présence du cadavre et l'horreur de la putréfaction qu'il suscite universellement constituent de puissants incitatifs à l'action rituelle. Malgré la diversité des solutions expérimentées par les humains des diverses époques et cultures, on peut regrouper sous trois grands invariants les moyens de disposer des cadavres : la destruction, la dissimulation ou la conservation ${ }^{93}$.

Par destruction du cadavre, on entend sa crémation pour le réduire à l'état de cendres. Par la suite, les cendres sont récupérées et traitées de diverses façons pour être conservées selon divers modes ou pour être dispersées selon des modalités aussi variables. L'autre forme attestée de destruction du cadavre est la manducation ou, dit autrement, le fait de manger le cadavre (endocannibalisme direct) ou de la faire manger par des bêtes qui seront à leur tour éventuellement mangées par les humains (endocannibalisme indirect).

Une autre option est de soustraire au regard des pairs la putréfaction que l'on ne peut envisager ou endurer. Trois possibilités ont été inventoriées par les anthropologues : l'inhumation du corps sous terre ou dans une grotte, l'immersion ou, finalement, l'isolement du cadavre dans un lieu éloigné ou inaccessible aux regards.

La dernière possibilité consiste à prendre les moyens pour conserver le corps en bloquant le processus naturel de la putréfaction de manière définitive ou temporaire. On a pu y arriver grâce au procédé de la momification, ou par les techniques de l'embaumement qui ne permettent toutefois que de retarder le processus que l'on cherche à éviter. D'autres cultures optent plutôt pour le «boucanage » du corps audessus d'un feu pour le déshydrater complètement et le conserver ensuite, bien en vue à la maison, en tant qu'ancêtre. Plus récemment, une quatrième technique a été développée, technique qui a pour nom la cryogénisation et qui consiste à surgeler le corps dans de l'azote liquide dès que possible après le décès dans l'espoir que la science pourra

\footnotetext{
92 Ou comme le dit Jean-Didier Urbain, L'archipel des morts, op. cit., p. 28 : il faut assurer le "contrôle organique des morts par les vivants".

"1bid., p. 28-33.
} 
remédier à son «mauvais fonctionnement » et le ressusciter le moment venu.

Ces différents types de dispositions pris à l'égard du cadavre ne sont pas mutuellement exclusifs et on peut souhaiter retenir une combinaison de ces méthodes. Ainsi l'on pourra incinérer le corps pour ensuite enterrer les cendres ou les disperser dans un cours d'eau ou ailleurs, ou encore les conserver dans une urne chez soi ou ailleurs. De la même manière, il est possible d'embaumer un corps pour ensuite procéder à son inhumation ou à sa crémation par exemple. Il est également possible de déterrer un cadavre après un temps déterminé (pratique souvent associée au rite des secondes funérailles) pour en récupérer les ossements que l'on lavera pour les conserver, par la suite, selon diverses modalités.

Les rites funéraires répondent donc ainsi et partout à une première fonction sociale qui est d'assurer le devenir du défunt en réglant d'abord le sort du corps mort qui n'est plus la personne aimée, mais qui est quand même plus qu'une chose inanimée. Cette première fonction du rite accomplie correctement favorisera normalement la réussite des deux autres, soit la prise en charge des endeuillés pour leur procurer une forme d'apaisement et la revitalisation de la communauté qui se recrée dans ce contexte de solidarité nécessaire entre les vivants qui passe par le respect envers ses morts.

2.7. Les rites à l'origine d'une culture funéraire matérielle universelle

Les morts se cherchent toujours une place, ils en ont besoin pour exister, et les vivants se doivent de leur en trouver une, aussi modeste ou discrète soit-elle, plutôt que de ne leur en procurer aucune ou de la leur refuser. La terre aux vivants? On ne congédie pas ainsi les morts, sans protocole ni cérémonie, sans lieu non plus. [...] aucune culture ne le fait $[\ldots]^{94}$.

Avec les rites funéraires, on se voit dans l'obligation de se séparer du cadavre sans s'en séparer totalement. Cette ambivalence des rites funéraires fait qu'ils nous amènent d'abord à nous séparer du corps mort (après un laps de temps plus ou moins long, selon les traditions et selon les soins apportés au corps de la personne décédée, ce que Thomas appelle la " retenue du corps") pour ensuite nous aider à lui 
trouver une nouvelle place permettant de le réintégrer au circuit de la vie des survivants et donc de conserver nos morts. Pour cela, toutes les sociétés ont mis sur pied une culture funéraire matérielle capable de conserver une trace des défunts, en leur assignant une nouvelle place, capable aussi d'alimenter le souvenir, la mémoire ou le culte des morts. «Toujours est-il que déjà le cadavre humain a suscité des émotions qui se sont socialisées en pratiques funéraires, et que cette conservation du cadavre implique une prolongation de vie. Le non-abandon des morts implique leur survie ${ }^{95} \%$.

Comme l'indique l'archéologue Jean-Pierre Mohen, l'observation, sur une aussi longue période que 100000 ans, de cette mosaïque planétaire des rites funéraires révèle bien des différences sociales et culturelles quant aux réactions des vivants face à la mort de l'un des leurs. Elle confirme pourtant aussi une certaine continuité de comportements propre à l'espèce humaine ${ }^{96}$. En effet, partout la mort est irruption du désordre qui doit s'accompagner, dans toutes les cultures, de gestes permettant la reprise du cours normal de la vie après la tenue de rites de passage adéquats ${ }^{97}$. Ces pratiques funéraires ne sont pas non plus nées par hasard. Elles sont reliées aux mythes fondateurs de chaque groupe et elles constituent les signes concrets de leur validation au quotidien ${ }^{98}$.

D'ailleurs, tous les rites funéraires comportent deux dimensions sociales distinctes : ils permettent à la fois l'individualisation et la socialisation. Dans le premier cas, le rite permet de reconnaître à chaque individu sa singularité. En effet, malgré les rites singularisants liés à sa naissance, malgré une particularisation liée à de rares événements au cours de sa vie, l'individu sera passé, sa vie durant, plutôt inaperçu. Dans le même mouvement pourtant, le rite funéraire contribue aussitôt à réintégrer le défunt dans son contexte social et à le faire participer et à l'inscrire dans ce courant, à cette lignée particulière et à ses valeurs. Ces deux dimensions du rite sont observables à partir de la culture funéraire matérielle, comme l'exprime bien le sociologue Claude Javeau.

Edgar Morin, L'bomme et la mort, op. cit., p. 33.

Q J Jean-Pierre Mohen, "Le propre de l'espèce humaine... ", op. cit., p. 320.

97 Martine Segalen, Rites et rituels contemporains, op. cit., p . 45.

ys Jean-Didier Urbain, L'archipel des morts, op. cit., p. 27. 
La dimension d'individualisation se lit dans le souci de conserver et préserver des restes distincts d'autres restes, quel que soit le traitement ayant été réservé à ceux-ci. Celle de la socialisation se manifeste dans le souci de faire figurer ces restes au rang des objets collectifs destinés à illustrer, de manière plus ou moins ostentatoire, les modalités les plus contraignantes du vivre-ensemble. Dans ce cas, il s'agit du "culte des morts", à travers les monuments ou autres signes de leur présence qui leur sont consactés. Les monuments individuels rassemblés dans les cimetières transforment ceux-ci en cité des morts, lesquelles entretiennent avec les cités des vivants des liens ambigus, dominés, selon des degrés divers, par le couple attraction-répulsion".

Chaque société, avec sa culture funéraire matérielle propre, affiche son profond désir de pérennité. «Momies, cendres, ossements sont en effet des substituts indestructibles, symboles d'immortalité qui nient la néantisation de la mort. Sauf exception, toutes les civilisations s'acharnent à les conserver et à les honorer ${ }^{100}$ ", d'où peut-être la phrase célèbre de Périclès dans la Grèce antique : «Un peuple se juge à la manière dont il ensevelit ses morts». Les cas où l'on refuse la sépulture à quelqu'un ou ceux où l'on s'en tient à l'utilisation de la fosse commune sont liés soit à une volonté de punir, soit à l'impossibilité d'agir autrement.

Différents auteurs ont examiné avec attention l'évolution récente de la crémation en Occident en lien avec ce désir de conservation des traces de nos morts. Jean-Didier Urbain ${ }^{101}$ a résumé le caractère à première vue ambivalent de ce choix en parlant du « pragmatisme crématiste » occidental par opposition à la métaphysique orientale à ce sujet. Si, en Orient, la symbolique du feu est associée à la purification et à la libération, la tradition occidentale symbolise plutôt la sanction (bûcher des sorcières, flammes de l'enfer) ou l'extermination (cadavres incendiés lors d'épidémies, destruction en temps de guerre, holocauste). Comment alors expliquer depuis peu la montée de la crémation en

"Claude Javeau, "Retour sur les rites piaculaires : pratiques et rôles dans l'immédiat et à distance ", Études sur la mort, n" 123, Morts et deuils collectifs, L'Esprit du temps, 2003, p. 72.

Lar Louis-Vincent Thomas, Rites de mort. Pour la paix des vilants, Paris, Fayard, 1985, p. 186-187.

101 Jean-Didier Urbain, "La cendre et la trace. Ja vogue de la crémation ", Frédéric Lenoir et Jean-Philippe de Tonnac (dir.), op. cit., p. 1207-1217. 
Occident? Pour Urbain, au-delà des arguments pragmatiques à caractère soit consumériste (c'est moins coûteux), soit écologique (c'est plus proche de la nature), soit esthétique (cela permet des rites plus dépouillés), soit individualiste (cela occasionne moins de soucis pour les proches), ou ceux qui se résumeraient à associer trop étroitement ces changements à la levée de l'interdit religieux chez les catholiques depuis 1963 , il faut plutôt chercher du côté de l'urbanisation de nos sociétés (l'incinération étant beaucoup plus pratiquée à la ville qu'à la campagne) et de la privatisation de notre rapport à la mort, qui grâce à la crémation permet la mobilité des restes et le repli vers l'intime ou des formes de sociabilité restreinte ${ }^{102}$.

[Les] cimetières se sont éloignés et avec eux les morts. Ils s'y égarent et se perdent en des lieux malgré tout assez vastes pour que la tombe y devienne un endroit anonyme, perçu comme immergé dans une multitude publique et fonctionnelle que domine le sentiment de surpopulation, de distance, de dissolution, de naufrage de l'individu et de désagrégation sociale des défunts ${ }^{103}$. Dans ce contexte, où le cimetière a perdu, avec sa proximité ct sa "taille humaine", It statut fédérateur de lieu communantaire central du travail de mémoire, d'album de famille collectif, la crémation apparait alors comme un moyen de le restaurer. De se réapproprier dans l'espace proche, avec la réduction cinéraire d'un défunt portatif libéré des pesanteurs physiques et administratives de l'inhumation traditionnelle, une qualité sociale et intime de relation aux morts annihilée par ces dispositifs collectifs trop gros et trop lointains que sont devenues les nécropoles périphériques des villes ${ }^{104}$.

Un peu plus loin, il poursuit :

[...] on soulignera que la frontière entre crémation et inhumation se trace moins désormais à partir d'oppositions religieuses ou idéologiques (entre protestants et catholiques, hygiènistes et traditionnalistes, athées et croyants ou libres penseurs et conformistes) qu'à partir de ces autres différences, entre ville et non-urbain ou public et privé... ${ }^{115}$

Ainsi, contrairement à ce qu'en ont dit plusieurs observateurs, Urbain relève que la crémation ne fonctionne pas seulement dans une logique

102 Ibid., p. 1211-1213.

10.3 D'après l'enquête Cif/Crédoc, de J.-P. Loisel et O. Martin, Les Français et le sounenir des morts, Paris, novembre 2000.

104 Jean-Didier Urbain, "La cendre et la trace. La vogue de la crémation ", op. cit., p. 1212.

tos Ihid., p. 1213. 
négative de destruction des restes, mais pourrait permettre à la fois la conservation des traces et le travail de mémoire, quoique par le biais d'un « nouveau pacte cultuel entre les vivants, les morts et la société ${ }^{106}$ », et ce, à un niveau plus microsocial que par le passé. En revanche, Urbain signale que cette nouvelle et très récente logique crématoire est en rupture avec une " philosophie de la mort sans trace ni lieu qui prônait sa dématérialisation ${ }^{107} »$, l'envers du phénomène étant que « [la] privatisation du culte entraîne une privatisation de la mémoire, et par l'émiettement de ses signes, la fin d'un passé partagé ${ }^{108} \gg$.

\subsection{Portée symbolique des rituels et leur impact}

Les rites constituent à la fois un espace et un système de communication. Ils donnent lieu à une utilisation intensive des symboles. Dans le cas particulier où la mort frappe près de nous, elle a pour effet de nous propulser dans un monde clos où ne subsiste en réalité qu'un cadavre. Les rites funéraires nous en font sortir en nous ouvrant les portes de la représentation symbolique et de l'imaginaire qui vont resituer la mort dans la vie. "La mort ainsi inscrite dans un cheminement, avec ses temps forts, ses douleurs et ses fêtes, garde sa violence, mais cesse d'être une absence pour devenir une dimension de la $v^{1 e^{109}}$ ». C'est donc seulement par la médiation de l'imaginaire qu'un changement de cadre est possible. D'un cadre social primaire, l'humain a accès, grâce au rite, à un cadre social transformé par le recours aux symboles. La crédibilité du rite tient d'ailleurs à sa capacité d'opérer ce changement de registre mental : " [...] le contexte rituel dans son ensemble va aller jusqu'à générer des états modifiés de conscience, la réalité devenant symbolique, et le symbolique transformatif, puisque capable de transformer cette réalité ${ }^{1 / 0}$ ». Marcel Mauss disait d'ailleurs

\footnotetext{
1 (or Ibid., p. 1214.

111 Ibid., p. 1214.

111 Ibid., p. 1214.

109 Roland Goetschel, «Tu retourneras à la poussière. Pratiques et rituels de la mort dans le judaisme ", dans Frédéric Lenoir et Jean-Philippe de Tonnac (dir.), Ia mort et l'immortalité. Encyclopédie des savoirs et des croyances, Paris, Bayard, 2004, p. 354.

111. Pascal Lardellier, Théorie du lien rituel, op. cit., p. 92.
} 
que les rites avaient la capacité de modifier l'état de la personne y participant et Victor Turner, que les symboles étaient les molécules du rituel. On peut aller jusqu'à dire que, dans le contexte rituel, tout est connotatif, c'est-à-dire que rien ne doit être pris ou interprété au premier degré, que rien n'est strictement «fonctionnel », mais que tout est fortement investi symboliquement. Pascal Lardellier est très clair à ce sujet.

La seule certitude, c'est que la signification est ailleurs que dans l'immédiateté et la platitude apparente de «ce qui se passe» sur la scène rituelle. Car la performance rituelle n'est pas la réalité, pas plus qu'elle n'est simplement un spectacle. Par-delà même sa dimension sémantique, c'est à l'efficacité symbolique, qui est sa raison d'être, que le rite appelle toujours ${ }^{\prime \prime \prime}$.

Le rite utilise donc des symboles, des signes collectifs, qui font sortir les participants de l'ordre usuel ordinaire, qu'il soit naturel, social, physique ou matériel. De là, le rituel a la capacité de les convier à une rencontre avec l'invisible, l'indicible, l'irreprésentable ou l'absent (à la condition que les participants soient là de leur plein gré et qu'ils rendent leur esprit disponible à ce qui va se dérouler sous leurs yeux). Il a le pouvoir de suppléer au manque de mots et de permettre, dans le cas d'une mortalité, l'expression et la «domestication »d'émotions fortes liées à une impuissance ressentie comme extrêmement menaçante. Loin de la logique instrumentale qui fait appel à la rationalité et au contrôle des émotions, le rite s'appuie sur une logique symbolique qui met ces émotions en scène au lieu de les refouler. L'utilisation des symboles tient de la représentation, donc d'une distance salutaire par rapport au vécu immédiat tellement douloureux et qui ne peut être maitrisé avec la raison ${ }^{112}$. Les rites ont préexisté aux religions et à la science. Ils ont été inventés pour répondre à un besoin primaire et primitif, celui d'entrer en contact, d'amadouer ou de maîtriser ce qui est puissant, numineux, mystérieux. L'humain contemporain voudrait peut-être se soustraire à ce besoin d'être réassuré quand il fait face à la mort et à la question de l'après, mais qu'il y soit parvenu, rien n'est moins sûr. Comme l'écrit Maurice Gruau, «[si] les hommes utilisent les rites depuis si longtemps, ce n'est pas nécessairement par une étrange faiblesse d'esprit mais peut-

Ibid., p. 110.

1!2 Denis Jeffrey, Éloge des rituels, op. cit., p. 18. 
être parce que certaines des réalités humaines ne se montrent pas facilement d'une autre manière ${ }^{113} »$. Et il poursuit en disant :

Or, c'est justement parce que les rites sont en partic irrationnels qu'ils ont des chances de durer. [... Il n'y a guère de lieux où il soit permis de dire sans dire, d'exprimer de manière tangente, ambiguë et équivoque, ce qui nous fait courir, ce qui nous motive et que nous ne savons pas bien'lt.

Parler du besoin du rituel funéraire et de sa pertinence, c'est rappeler que le rôle du rite est d'abord de prendre acte de la brutalité d'une réalité, la mort, et ensuite de répondre au non-sens de cette mort, à cette rupture et à cette provocation qui ne peuvent être ignorées ni par l'endeuillé, ni par son groupe social. Culturellement, on ne peut rester coi mais, individuellement, on est sans mot. Devant le choc, l'absurdité, le vide et la violence - particulièrement la violence symbolique - de la mort, l'humain reste sans voix, presque sans vie littéralement. Position intenable qui appelle un mouvement, une réaction, une recréation symbolique par l'intermédiaire du social et donc de la communication. Le rituel funéraire, dans sa fonction première, « [...] harmonise la vie et la mort dans une fragile tension ${ }^{115} »$. C'est en ce sens que le rite reconnaît que la personne est morte et, en même temps, qu'elle n'est pas morte et qu'elle est en transformation vers un autre mode de vie ${ }^{116}$. Or, les rites sont justement une forme complexe et complète de communication. En effet, en dehors de la communication dans son acceptation la plus large, les rites funéraires ne sont rien. En fait, les rites sont communication, essentiellement. Communication verbale, non verbale, symbolique, physique. Tentative aussi probablement de communication avec une transcendance, toujours par la médiation symbolique. Cette façon de ruser avec la mort - de faire passer le drame du plan réel au plan symbolique - «fonctionne» depuis cent millénaires malgré les multiples formes empruntées au fil du temps.

Les symboles utilisés doivent par contre être collectifs et reliés aux mythes et récits fondateurs pour avoir un pouvoir évocateur suffisant.

11.3 Maurice Gruau, L'bomme rituel. Anthropologie du rituel catbolique francais, Paris, Métailié, 1999 , p. 16.

it Ibid., p. 213.

115 Denis Jeffrey, Jouissance du sacré, op. cit., p. 149.

1" Isabelle Richard, "Mourir à l'hôpital », dans Marie-Frédérique Bacqué (dir.), Mourir aujourd'bui. Les nouveaux rites funéraires, Paris, Odile Jacob, 1997, p. 130. 
En effet, un symbole n'est jamais personnel. C'est pourquoi, pour produire le sens dont les participants au rituel ont tellement besoin, les ressources de l'imaginaire collectif sont seules susceptibles d'y arriver. Le rite utilise aussi un support discursif et narratif, mais ce dernier à lui seul ne parvient pas à nous faire sortir du cadre social habituel pour nous faire entrer dans le cadre transformé d'un espace social où les règles habituelles ne tiennent plus tout à fait et où même la notion du temps n'est plus la même. Marie-Frédérique Bacqué renchérit sur ce point en disant que « [le] rite permet un ralentissement autour de l'événement qui ratifie un état de fait que nous refusons de toutes nos forces ${ }^{17}$ ». Luce Des Aulniers, en parlant de notre époque, dit au sujet du temps rituel : «[...] nous n'avons pas de temps pour le rite, parce que nous n'avons pas le temps de faire place au temps dans lequel nous fait entrer tout rite ${ }^{118} »$. Seuls ce temps modifié, de même que le contenu et la grammaire symboliques du rite - assistés, il faut le dire, par des moyens techniques et une mise en scène adéquate - transmis comme un héritage à travers les générations ont la capacité de magnifier et d'impressionner, selon les mots de Lardellier, les situations qui dépassent notre entendement et de créer un pont pacificateur entre le social - le visible - et le psychisme - l'invisible.

Parler de la mort, c'est la faire vivre, c'est lui donner une réalité, c'est la sortir du grand vide. Pour parler de la mort, il faut des signes.

A force de produire et de reproduire, rituellement, des signes y référant - symboles pourtant "plus réels que ce qu'ils symbolisent » [comme l'a dit Jćvi-Strauss] - un « arrière-monde» (comme préférait dire Nietzsche) se met à exister, pour chacun et chez tous : aux confins de l'esprit, un au-delà s'inscrit, plein et pensable, peuplé, quasi familier, dans le prolongement du monde des vivants. Entre celui-ci et le monde des morts, une continuité de rêve se laisse concevoir, même confusément. [...]

Dans cette perspective, parlcr de la mort est une invention : c'est inventer de l'être contre du néant. [...] L'énonciation répétée des mots, la prière, la reproduction des gestes, la contemplation des images, la lecture des textes et la permanence des monuments, tout cela construit le rituel autour de la mort. La rêverie s'accomplit, s'ordonne, devient structure mentale, schèmc idéologique, institution, croyance, fondation d'une

11- Marie-Frédérique Bacqué, "Introduction. Retrouver l'émotion ", dans MarieFrédérique Bacqué (dir.), Mourir aujourd'bui, op. cit., p. 13.

11* Juce Des $A$ ulniers, «Bruit du temps jusqu’à silence de mort», dans Marie-Frédérique Bacqué (dir.), op. cit., p. 208. 
culture, code fondamental.

Dès lors, ainsi codée, la mort n'est plus étrangère, n'est plus l'Autre absolu" ".".

C'est ce qui a fait dire à Patrick Baudry, que « [dans] le rituel, il s'agit de placer un tiers, le tiers d'une culture, entre la mort et $\operatorname{soi}^{120} "$ pour servir d'intermédiaire dans une relation qui ne peut être directe avec l'impensable.

\subsection{Ce que les rites révèlent de notre rapport à la mort}

Anthropologues, historiens, spécialistes des sciences des religions et sociologues ont discerné à travers les âges des attitudes diverses à l'égard de la mort. Chaque période, chaque culture, chaque système religieux se caractérise par une perception de ce qu'est une «bonne mort», une « mauvaise mort » et, plus récemment, une «belle mort». De plus, il ne faut guère s'en surprendre, il est possible de trouver dans l'histoire des inversions parfaites qui font que les définitions de bonne et de mauvaise mort finissent par coïncider pour des groupes différents. Sans vouloir reprendre ici l'ensemble de ces travaux ${ }^{121}$, retenons que la transformation de nos pratiques rituelles funéraires est parallèle à l'évolution de nos rapports à la mort qui sont liés, à leur tour, à l'évolution de nos croyances et aux autres tendances lourdes traversant la vie des sociétés en général.

Quoique ces regards de chercheurs sur l'évolution de nos sentiments face à la mort empruntent des voies différentes, tous se rejoignent autour d'un même constat : progressivement, sur plusieurs siècles, la perception qu'a l'humain de la mort s'est modifiée assez profondément. Si la mort a pu paraître à nos ancêtres comme une force sauvage, aveugle, mystérieuse et terrifiante, elle est aussi devenue, avec le temps et le travail des rites, plus familière et civilisée - au sens de faisant partie d'un ordre connu et contenu. Si la mort menace d'abord, à l'origine, la stabilité du groupe social lui-même, petit à petit, c'est de plus en plus

\footnotetext{
11" Jean-Didier Urbain, L'archipel des morts, op. cit., p. 37.

12" Patrick Baudry cité par Maric-Frédérique Bacqué, "Introduction. Retrouver l'émotion ", op. cit., p. 13.

121 Dont les plus classiques sont ceux d'Edgar Morin, de Philippe Ariès, de LouisVincent Thomas, de Michel Vovelle et de Jean Zicgler.
} 
l'individu qui se sent concerné par sa propre disparition. Puis, au-delà de ce drame personnel, on s'attarde sur l'arrachement que la mort impose par rapport à ceux qu'on aime et qui meurent. Encore un pas et la mort est refoulée, niée et occultée, redevenue force indomptée et innommable. L'humain moderne a dorénavant affaire à un tabou. Ce tabou sera bientôt étudié, débusqué, critiqué, et nous voilà aujourd'hui en face d'une mort aux contours encore mal définis, mais que le corps social a réinvesti en la travestissant en mort bavarde - au sens de bruyante sur la place publique mais surtout scientifique - et en mort bricolée - au sens où son statut oscille encore entre une mort redécouverte et une mort fragmentée ou éclatée dans sa signification collective $^{122}$.

Patrick Baudry résume en deux paragraphes notre rapport actuel à la mort.

Nous avons à faire face à plusieurs difficultés. La difficulté à comprendre que la mort est une limite : plutôt la traite-t-on comme une frontière qui autoriserait des allers-retours. La difficulté à situer l'au-delà comme symbolique : plutôt voudrait-on s'en saisir comme d'un territoire. La difficulté à entrevoir la mort comme dimension qui marque l'existence humaine, alors qu'on en retient surtout l'aspect événementicl et donc individuel. Enfin, la difficulté à s'affronter au réel de la mort comme souffrance : plutôt a-t-on tendance à en traiter comme d'une disparition. ${ }^{123}$

Au fond, ce n'est pas la peur de la mort qui nous caractérise, mais la peur de cettc peur, c'est moins le refus du trépas que le refus de ce refus. Autrement dit, l'incapacité ou la difficulté à faire de la crainte le ressort d'une solidarité communautaire, ou à mettre en scène cette solidarité dans l'élaboration collective des peurs partagées. Ainsi, l'utilité profonde du rituel funéraire apparaît-elle clairement : il permet l'expression dirigée de l'angoisse de mort, en favorisant l'émergence d'un sens, en situant la mort à sa place, et en affirmant la vie ${ }^{124}$.

Entre la mort constatée au quotidien, la mort contestée dans sa réalité même et l'encore hypothétique et timide résurgence de la mort, nos

127 Nous mettons ici en garde le lecteur contre la fausse impression de linéarité qui se dégage de ce tableau récapitulatif de l'évolution de notre rapport à la mort. Bien entendu, les hésitations et les chevauchements de l'histoire sont occultés dans ce genre d'exercice, de mème que les importantes variations géographiques et culturelles.

12.3 Patrick Baudry, «Le sens de la ritualité funéraire », dans Marie-Frédérique Bacqué (dir.), Mourir aujourd'bui. Les nomeaux rites funéraires, Paris, Odile Jacob, 1997, p. 228.

$12+$ Patrick Baudry, «Le sens de la ritualité funéraire », op. cit., p. 229. 
sociétés, nos croyances, nos rites, notre coeur et notre raison balancent. Du feu qui couve sous la force des traditions, au brülis initié par la modernité et à la grisaille des cendres récoltées par la postmodernité, ily a continuité tout autant que rupture, disions-nous plus tôt.

2.10. Évolution des rites funéraires sous la pression de la modernité

Dans la brève histoire du développement des études sur la mort, au moins une controverse importante doit être signalée ici. Jean-Marc Larouche $^{125}$ signale que des auteurs comme Jean-Claude Chamboredon et Pierre-Philippe Druet critiquent le fait que les travaux de nombreux auteurs du champ des études sur la mort comme ceux d'Ariès, de Baudrillard, de Ziegler, de Thomas, de Morin, de Kübler-Ross et de Gorer présentent une certaine confusion des genres entre l'analyse et la déploration. Larouche retient, par exemple, ces propos de Chamboredon :

[...] les discours savants sur la mort contemporaine, tels ceux d'Ariès, Baudrillard et Ziegler, ne sont pas déliés d’une certaine déploration de la perte de sens de la mort et d'une "nostalgie du paradis social " perdu faites au nom " [d'un] traditionalisme passéiste, [d'un] conservatisme moral ou d'un anticapitalisme radical ${ }^{126}$.

Quant à Pierre-Philippe Druet, voici un extrait de son analyse que Larouche fait ressortir comme contribution à ce débat :

[...] presque tous les ouvrages récents combinent la spéculation philosophique et l'enquête empirique, selon des dosages divers. Leurs auteurs se déclarent adversaires du déni et entreprennent, qui de prouver son existence (Ariès, dans certains articles), qui d'en définir les causes (J. Ziegler, L.-V. Thomas), qui d'eh recenser et d'en combattre les effets (les "psychologues de la mort"). Le propos n'est que très rarement descriptif et l'apport des sciences humaines se trouve souvent "exploité " à des fins philosophiques et idéologiques ${ }^{127}$.

125 Jean-Marc Jarouche, "La mort et le mourir, d'hier à aujourd'hui », Religiologiques, n" 4, automne 1991, p. 1-41.

126 Jean-Claude Chamboredon, "La restauration de la mort. Objets scientifiques et phantasmes sociaux ", Actes de la recherche en sciences sociales, vol. 2, n" 2, 1976, p. 86, cité par Jean-Marc Jarouche, «La mort et le mourir, d'hier à aujourd'hui », op. cit., p. 2-3.

12- Pierre-Philippe Druet, Pourviure sa mort, Ars Moriendi, Paris, Éditions Lethielleux, 1981, p. 28, cité par Jean-Marc Larouche, "La mort ct le mourir, d'hier à aujourd'hui », op. cit., p. 24. 
Larouche se joint donc à ces auteurs et reproche à plusieurs pionniers de la thanatologie d'avoir présenté la mort et le mourir contemporains comme étant en opposition parfaitement symétrique au modèle de la mort d'autrefois et d'avoir ainsi contribué à ignorer le sens réel de certaines pratiques contemporaines ${ }^{128}$. En concluant son propos, il affirme, au sujet du mouvement thanatologique :

Ce mouvement émerge donc au gré d'un processus de mauvaise conscience et de culpabilisation devant les formes jugées aliénantes de la mort et du mourir dans la société moderne. Iaa visée de ce mouvement est de pouvoir susciter une réforme des représentations, attitudes et pratiques envers la mort et les mourants. Ce qui est en jeu, n'est rien d'autre que la manipulation symbolique. Jes acteurs qui participent à ce mouvement tentent en effet de redéfinir la symbolique et la normativité relatives à la mort et au mouriri"?.

Larouche reproche, en somme, au mouvement thanatologique son recours à la spéculation philosophique et d'avoir laissé penser qu'il y avait peut-être, par rapport à la mort, une bonne attitude à adopter pour le mourant et pour son entourage, une bonne façon de mener les rites funéraires, une bonne façon de faire son deuil, etc. Il leur reproche donc d'avoir établi quelque chose de normatif dans notre rapport général à la mort et d'avoir ainsi laissé entendre que ce qui s'éloignait trop de ce modèle normatif devait être revu et corrigé.

Ce genre de glissement de l'analyse vers le jugement de valeurs constitue un vieux problème des sciences humaines et un écueil qu'il n'est pas toujours facile d'éviter. Il faut avouer que le domaine de la recherche sur les rituels pose des défis particulièrement difficiles à relever parce que les rites, même si on peut les observer partiellement de l'extérieur, relèvent aussi bien de l'intériorité du sujet, en ce qu'ils sont connectés aux mythes, aux croyances, aux valeurs, aux attitudes et aux perceptions. S'ajoute à cela le fait déjà mentionné que la mort constitue le problème philosophique et social par excellence et qu'en ce sens, chercheurs, penseurs et lecteurs des productions écrites dans ce domaine d'études peuvent difficilement éviter d'être touchés personnellement par les enjeux sous-jacents à toute cette réflexion.

En revanche, un autre auteur, Patrick Baudry, pense que les travaux

\footnotetext{
12. Jean-Marc Larouche, «La mort et le mourir, d'hier à aujourd'hui », op. cit., p. 10.

129. Ibid., p. 34.
} 
de certains pionniers comme Thomas, Ziegler et Baudrillard ont pu éviter ce genre de malentendus.

On ne pouvait comprendre qu'ils situaient une origine vers laquelle nous devrions retourner, comme s'il était possible d'en décider psychologiquement. Ils faisaient des sociétés "primitives" non pas le lieu d'une sagesse exemplaire, mais le milieu d'une critique radicale des rapports contemporains à la mort : en révélant des inégalités, en refusant une cxploitation et en dévoilant une crise, ils mettaient globalement en cause un système de domination. Ces trois auteurs ne jugeaient pas seulement d'une mauvaise gestion de la "fin de vie ", mais d'une incapacité à comprendre que la mort ne se réduit pas à la cessation individuelle des fonctions vitales. Ce qui intéresse les fondateurs de cette approche critique, c'est la place de la mort dans les rapports sociaux et les solidarités complexes qu'elle provoque. [...] C'est aussi l'énigme de la mort, la limite qu'elle trace à la toute-puissance et le rappel qu'elle impose d'une vulnérabilité, qui sont soulignés ${ }^{131}$.

Il poursuit sa réflexion ainsi pour caractériser les travaux à mener dans ce domaine au XXI ${ }^{e}$ siècle.

La réflexion critique sur la mort prend ainsi la forme d'un combat. Eille n'a pas la douceur ou la naïveté d'un humanisme, ainsi que l'on pourrait l'y réduire.

L'objet du discours des sciences humaines n'est donc pas de donner la main à une société gestionnaire mais de contester une production de la vie dont le monde occidental croit détenir la bonne définition, et cela en rappelant notamment unc certaine expérience humaine que les sociétés dominées peuvent enseigner. Bien plus que la peur et l'angoisse (qui sont universelles et que les sociétés dites traditionnelles mettent en scène en ćlaborant un rapport collectif à la mort et aux défunts), c'est un déni de la mort « dans le réel » qui marque la modernité. Il ne s'agit plus d'un déni "symbolique " qui permet de mettre la mort à distance et à sa place. Dépossédée de la mise en scène rituelle et du travail qui s'y fait de l'imaginaire dans son articulation au symbolique, la société qui ne veut "penser qu'à la vie " doit s'astreindre à une esquive permanente de la finitude. Répétons-le, le déni n'est pas qu’un refus. Il provoque le double jeu de la répulsion et de la fascination ${ }^{1,31}$.

Pour lui, la question de la mort façonne littéralement notre rapport au monde et façonne l'institution même de la société. Dans le cas de la société contemporaine, ce qui pose problème, selon la thanatologie, ce n'est pas qu'elle se détourne de la mort - toutes les sociétés le font

1.31 Patrick Baudry, Paradoxes contemporains, op. cit., p. 893-894.

131 Ibid., p. 897. 
symboliquement - c'est qu'elle prétend l'oublier. C'est donc l'inconnu de la mort et le fait qu'elle appartienne à la vie - en tant que son contraire - qu'il convient de réaffirmer à une société qui voudrait s'en croire protégée par des cloisons étanches.

Les auteurs spécialisés dans les études sur la mort ont tous tenté d'expliquer l'évolution de nos rapports à la mort et aux rites en reliant ces phénomènes à un portrait d'ensemble de l'évolution récente des sociétés. Pour ce faire, les sciences sociales utilisent généralement les notions de tradition, de modernité et, plus récemment, de postmodernité et nous y reviendrons. Mais c'est Louis-Vincent Thomas qui a probablement consacré le plus de place dans ses ouvrages à décrire ce qui est advenu des rites funéraires en Occident. Pour lui, on peut résumer ces changements en deux temps à l'aide de quelques mots clés $^{132}$ : disparition, simplification, privatisation, technicisation, professionnalisation, changement des lieux, dissimulation, réduction, désocialisation et désymbolisation, puis prise en charge du survivant, personnalisation, participation, invention de gestes expressifs, médicalisation du deuil contemporain et, finalement, laïcisation des funérailles.

Disparition, parce que, au plan des rites, plusieurs composantes ou étapes du rituel traditionnel n'existent tout simplement plus : accompagnement du mourant et veille à la maison, toilette funéraire accomplie par des membres de la famille, cortège funèbre du lieu de culte jusqu'au cimetière, rites de deuil - à peu près dans leur entièreté - et commémoration publique du décès dans les mois ou les années qui suivent.

Simplification parce que, d'une manière générale, le caractère plus formel ou solennel des rites a été abandonné ou grandement simplifié, à preuve la disparition de tous les éléments précédemment cités et l'apparition d'un menu davantage à la carte où les choix se multiplient et présentent davantage de souplesse qu'auparavant. Il est dorénavant possible d'expédier en 24 heures l'ensemble du processus redéfini aux

132 Cet aspect de l'œuvre de J.ouis-Vincent Thomas est abordé de façon succincte dans La mort, Paris, PUF, [1998], 2003, p. 102-106, mais ce même thème est développé davantage dans plusieurs autres ouvrages dont Rites de mort. Pour la paix des vivants, op. cit., p. 51-112 et dans un extrait d'un article d'abord paru sous le titre « Problèmes actuels de la mort en Occident ", dans Prétentaine, Université de Montpellier 3, 1994, p. 95-107 et repris sous le titre « Iouis-Vincent Thomas ou l'Occident revisité », dans Frontières, vol. 11, n" 1, $10^{c}$ anniversaire, 1998, p. 15-20. 
goûts et aux besoins du jour comme le proposent explicitement certaines entreprises nord-américaines ou comme le pratiquent sur demande la plupart des entreprises.

Privatisation en deux sens. D'abord parce que les individus et les familles ont de plus en plus d'occasions de personnaliser les rites funéraires pour tenir compte de l'individu visé par ces rites, de ses dernières volontés, de sa vie, de ses croyances ou de sa non-croyance, des liens qu'il a établi avec son entourage et avec sa communauté. Mais privatisation également dans le sens où les rites sont moins qu'avant l'occasion d'un large rassemblement communautaire quasi obligatoire ou automatique. Le groupe de personnes rejoint compte ceux qui, sauf exception dans le cas des décès de personnalités publiques, ont vraiment ou ont davantage côtoyé la personne défunte.

Technicisation, bien sûr, en raison de l'apparition et de la généralisation des techniques de la thanatopraxie qui ont révolutionné les pratiques - et la traditionnelle et quasi universelle toilette des morts qui était davantage un acte de purification et de respect qu'un acte hygiénique ou de maquillage du corps mort -, mais probablement aussi toute la symbolique entourant la rencontre avec le cadavre. On ne présente pas le plus souvent un corps inanimé, diminué, amoindri ou méconnaissable en raison de la maladie ou brisé par un accident, mais un corps " revampé ", plus digne et qui ne suscitera ni l'horreur ni la peur ni le rejet. Un corps qui n'a rien d'un cadavre, mais qui a plutôt l'air de dormir ou de se reposer (un «beau mort»), tellement qu'il donne parfois l'impression d'être sur le point d'ouvrir les yeux et de se réveiller. Le travail de deuil peut ainsi commencer plus sereinement, dit-on. Technicisation aussi par le biais de la crémation qui prend de plus en plus de place - et dont on pourrait analyser les idéologies légitimatrices - et qui est à des lieues de la crémation en plein air, sur un bûcher, encore pratiqué à d'autres endroits de la planète ou en d'autres temps en Amérique même par certaines tribus amérindiennes. Crémation qui n'apparaissait pas alors comme un geste technique pratiqué par des employés utilisant une technologie de pointe qui en moins d'une heure réduira en cendres, c'est le cas de le dire, un corps humain, quitte à passer les résidus dans un broyeur pour obtenir un produit plus uniforme. L'absence totale non seulement de la richesse symbolique des crémations traditionnelles en lien avec les mythes fondateurs d'un groupe, mais même d'une quelconque préoccupation 
en ce sens, caractérise nos nouvelles pratiques. On mise sur l'efficacité, la rapidité d'exécution, la réduction des coûts et le caractère hygiénique et écologique de l'opération.

Professionnalisation en lien avec l'apparition de l'entrepreneur funéraire et de son équipe, de leurs contrats, de leurs produits et services, de leurs locaux pour les vivants comme pour les morts, de leur guichet unique et de leurs techniques modernes. Voilà combien d'atouts réels, mais au prix de la disparition ou de l'éclipse des rapports humains de proximité et d'intimité pour plusieurs moments du rite. Professionnalisation aussi, rappelons-le, par le biais du séjour à l'hôpital où malade et membres de son entourage se retrouvent " reçus » dans un lieu qui leur garantit un maximum d'efficacité et de sécurité par rapport à la maladie et à la mort devenues de plus en plus étrangères pour nos contemporains, mais qui se retrouvent « invités » dans un lieu qui n'est pas le leur et qui possède ses propres normes et règles de conduite (horaires, places et traitements disponibles, aspects légaux des décisions à prendre, etc.).

Changement des lieux justement avec l'hôpital et le complexe funéraire qui prennent le relais de la maison du mourant et de l'église elle-même pour un nombre grandissant de cas. Changement aussi de lieux parce que les convois funéraires collectifs n'existent plus pour déplacer le cadavre vers son lieu de sépulture. Changement des lieux finalement parce que la pratique de la dispersion des cendres multiplie tout autant qu'elle efface les lieux retenant les traces de notre passage.

Dissimulation parce que tout est entrepris d'abord pour que le mort n'ait pas l'air d'un mort, mais d'un vivant endormi - si toutefois il est même exposé, auquel cas la dissimulation est alors totale -, et ensuite pour que les cimetières s'intègrent tellement bien au paysage de nos villes qu'on ne les reconnaisse plus comme tels : cimetières-parcs, cimetières-tours (le cimetière est tout simplement un édifice en hauteur où chaque étage voit s'aligner enfeux et niches de columbarium un peu comme les tours de logement font que les voisins habitent des appartements adjacents) et cimetières forestiers (où les morts reposent au pied des arbres, en retrait des villes et cachés au regard des passants).

Réduction parce qu'on assiste à une logique où toutes les traces traditionnelles de la mort et des morts sont ramenées à leur plus simple expression, en commençant par le cadavre réduit en cendres, aux inscriptions minuscules figurant sur les niches des columbariums et aux inscriptions à tendance minimaliste que certaines études ont permis 
d'observer de plus en plus sur les épitaphes : comme par exemple d'y inscrire tout simplement « Mère » sans plus.

Désocialisation aussi des rites funéraires qui, on le voit à la lecture des paragraphes précédents, perdent progressivement leur capacité de rassemblement pour faire de l'événement de la mort une occasion de renouer les liens sociaux devant ce mystère et son pouvoir de néantisation. C'est le contraire qu'on observe : simplification importante de ce qui subsiste des rites, funérailles réunissant de faibles foules, endeuillés abandonnés socialement au lendemain des funérailles. Luce Des Aulniers ajoute, en renfort à cette analyse, que lorsque l'on entend que tout en matière de funérailles est affaire de choix personnel, on a justement là le signe patent d'une désocialisation de ces pratiques ${ }^{13.3}$.

Désymbolisation alors que tout le pouvoir évocateur et guérisseur des rites repose théoriquement sur la pertinence, la justesse et la puissance des symboles utilisés et connectés en droite ligne avec nos mythes ou récits fondateurs, à peu près tout dans l'évolution récente des rites funéraires contribue à achever cette désymbolisation, en commençant par la crémation qui, dans nos cultures, est totalement dépourvue de ce recours aux symboles. L'exception à ce qui semble devenu une tendarce lourde vers la désymbolisation repose sur les expériences de «personnalisation » des funérailles, que ce soit dans les funérailles religieuses ou civiles.

Toujours selon Thomas, après une période qui laissait croire à l'obsolescence progressive des rites funéraires, on assiste en même temps, au cours des trois dernières décennies, à une certaine renaissance des rites. Sans parler de restauration des anciens rituels, on observerait une tendance à l'innovation rituelle.

Cet intérêt nouveau passe d'abord par ce qu'il nomme la prise en charge des survivants. On se soucie dorénavant beaucoup plus d'accompagner les endeuillés dans cette période difficile, par l'entremise des rituels funéraires, que de maintenir, au premier plan des préoccupations rituelles, le devenir spirituel du défunt.

Thomas parle également de personnalisation des funérailles. Les responsables du culte, de même que les employés des maisons funéraires, veillent à préparer des cérémonies qui tiennent compte de la

\footnotetext{
13.3 Luce Des Aulniers, "Bruit du temps jusqu’à silence de mort », op. cit., p. 218.
} 
personnalité du défunt, de sa vie et des circonstances de son décès. Par ailleurs, le défunt lui-même énonce de plus en plus souvent ce qu'il souhaite pour que ses funérailles soient à son goût et les familles des personnes mortes investissent temps, efforts, consultations et négociations pour arriver à organiser et à vivre des rites qui soient les plus significatifs possible pour eux et le plus possible à l'image du disparu. Ce « renouvellement » ou cette « réinvention » des rituels correspond bien au puissant phénomène d'individualisation typique de notre société, et même l'Église catholique, dans sa réforme des rituels, a prévu laisser une place à l'expression de ces souhaits de personnalisation des funérailles.

Une autre tendance récente, très liée à la précédente, a trait à la participation pendant les funérailles. Le statut traditionnel de «spectateur passif » de ceux et celles qui assistent aux rites est mis de côté pour leur permettre de s'engager dans la préparation et le déroulement de la cérémonie. On cherche ainsi à donner la parole aux familiers qui, de toute façon, veulent faire quelque chose.

Sur le plan des symboles associés à la ritualité funéraire, on chercherait de plus en plus à permettre la recherche et linvention de gestes expressifs qui font là encore référence à l'histoire de vie de chaque personne décédée et des membres de son entourage. On souhaite trouver des composantes symboliques plus significatives et adaptées à chaque histoire. Ainsi, on parle moins de glissement vers la déritualisation, comme la période précédente le laissait entrevoir, mais plutôt d'une ritualité nouvelle, "plus humaine mais moins spirituelle ».

Avec la médicalisation du deuil contemporain, c'est un autre aspect de la ritualité funéraire qui est visé. L'objectif est de sortir l'endeuillé de sa solitude après les funérailles. Comme la mort a été médicalisée en passant par l'institution hospitalière, le deuil fait l'objet d'un intérêt croissant de spécialistes, professionnels ou bénévoles formés, chargés d'aider la personne à « réussir son deuil », mais en dehors des voies plus communautaires du rite traditionnel.

Finalement, Thomas voit dans ces tendances nouvelles l'expression d'une laïcisation des funérailles. En effet, toute la vie rituelle est axée sur l'existence concrète de celui qui a vécu et de celui qui reste. 
Concernant la thématique de cette renaissance des rituels, JeanClaude Besanceney ${ }^{1.34}$ a tenté d'en cerner les principales tendances, tout en avouant le manque d'enquêtes à ce sujet. Il y décèle les caractéristiques suivantes :

- une volonté affirmée des proches de faire quelque chose (ce qui sous-entend souvent de faire autre chose que ce à quoi on nous a habitués) ;

- une forte demande et recherche de conseils à propos de funérailles significatives ;

- l'absence de l'expression d'une symbolique quant à la destinée postmortem et une insistance accordée à la consolation des vivants ;

- le fait que ces recherches rituelles ne sont pas reçues par transmission comme c'était le cas auparavant ;

- un déplacement de la fonction sociale des entreprises funéraires qui changent de statut en devenant de véritables promoteurs responsables de l'ensemble des funérailles;

- un éclatement de l'espace rituel qui ne se borne plus à l'église ;

- l'absence de référence à la transcendance et même l'absence de la dimension de la vie spirituelle.

En terminant cette section, ajoutons que le développement de ces « nouveaux rites » a aussi entrainé des questionnements à savoir s'ils constituaient véritablement des rites funéraires à part entière. Luce Des Aulniers a d'ailleurs proposé trois balises pour nous aider à en juger ${ }^{135}$. Pour elle, le rite funéraire doit d'abord reconnaitre que la mort est une malédiction, un déchirement, un arrachement. Ce n'est qu'ensuite qu'on peut espérer obtenir une forme d'apaisement pour les endeuillés; on ne peut donc faire l'économie de l'aveu que la mort est un sale coup. Ensuite, le rite funéraire s'inscrit toujours dans la matérialité du corps, c'est-à-dire qu'il doit permettre à la réalité de la mort de pénétrer la personne autrement que par sa raison uniquement : par exemple par le biais des démarches bien terre à terre que l'on doit faire avant la tenue des rites, pour les organiser, ou par le biais des gestes physiques que l'on

1.34 Jean-Claude Besanceney, "Évolution des rites catholiques du deuil et nouvelles pratiques rituelles", dans Marie-Frédérique Bacqué (dir.), op. cit., p. 189-192.

13. Luce Des Aulniers, “Bruit du temps jusqu’à silence de mort», dans Marie-Frédérique Bacqué (dir.), op. cit., p. 215-219. 


\section{NPSS, VOLUME 3, NUMERO 1, 2007}

pose durant la cérémonie. Finalement, le rite s'inscrit dans une culture. Il ne peut être réduit à l'éloge du lien personnel qui unissait chacun au défunt. L'individu et le social ne peuvent se tourner le dos au moment du rite.

\subsection{Sur les notions de modernité et de postmodernité}

Comme notre objectif ici n'est pas de faire état du travail ayant donné naissance au concept de postmodernité, mais d'interpréter l'évolution des rites funéraires actuels à la lumière de ce nouveau contexte socioculturel, nous rappellerons simplement que nous entendons par postmodernité " l'hypothèse globale nommant le passage lent et complexe à un nouveau type de société, de culture et d'individu naissant au sein même et dans le prolongement de l'ère moderne ${ }^{136}$ ». La postmodernité est donc une recomposition historique et sociale parce qu'on ne réinvente pas la roue et que l'horizon de la pensée demeure ici la modernité. C'est pourquoi Maffesoli parle d'un processus sociohistorique de "saturation-recomposition ». Dans la même veine, Yves Boisvert ${ }^{137}$ introduit une autre donnée intéressante pour distinguer modernité et postmodernité. Selon lui, la postmodernité doit être étudiée en termes de «mutation " plutôt qu'en termes de " révolution ", comme on l'a fait pour analyser le passage de la tradition à la modernité. En effet, le concept de révolution évoque une logique de rupture avec le passé, en particulier une rupture «consciente» et délibérée sur le plan de l'ancrage économique - mais pas exclusivement - entre les deux formes d'organisation sociale. Les sociétés de la tradition étaient des sociétés d'autosuffisance axées au départ sur la chasse et la cueillette, puis sur l'agriculture et le travail de l'artisan, alors que les sociétés de la modernité ont été marquées du sceau de la production de masse de nature industrielle, grâce à l'accumulation du capital, aux nouveaux outils de travail et à l'organisation du travail qu'on a pu y déployer. Par contraste, pour le passage à la postmodernité, une mutation suit plutôt une logique de transformation «non intentionnelle » qui s'opère dans le

13̆ Gilles Lipovetsky, L'ère du vide, Paris, Gallimard, 1983, p. 114.

13. Yves Boisvert, "L'analyse postmoderniste au-delà de l'esthétisme discursif ", dans

Yves Boisvert (dir.), op. cit., p. 181-183. 
prolongement de la modernité, surtout en ce qui concerne son ancrage culturel cette fois.

Nous nous appuierons ici surtout sur le sociologue Maffesoli ${ }^{138}$ pour résumer, très succinctement, et comparer plus avant modernité et postmodernité, essentiellement dans le but de comprendre ensuite les principales « mutations » à l'oeuvre dans notre monde et d'en mesurer l'impact sur notre rapport à la mort et aux rites.

Si la modernité repose, selon lui, sur l'État-nation, de nouvelles institutions sociales et un nouveau système idéologique, et se caractérise par un processus général d'homogénéisation sociale, qu'en est-il de la postmodernité ? Pour Maffesoli, toujours, elle s'appuie, parallèlement, sur trois piliers : le « retour du local », «l'importance de la tribu » et le "bricolage mythologique».

Par le « retour du local », il entend la nouvelle tendance à l'hétérogénéisation ou à la différenciation dans plusieurs facettes de nos organisations sociales. Le "localisme" revient à la mode. Selon son expression, "le lieu fait lien ». Ce qui lie socialement les individus doit laisser place à l'expression d'un " partage émotionnel » fondé sur la possession commune de valeurs de proximité (langue, coutumes, cuisine, etc.) et ne pas être qu'abstrait, théorique ou basé sur un idéal lointain.

Dans le même esprit, "l'importance de la tribu " réfère à cette exigence de proximité des liens et des valeurs, proximité qui manque justement dans les grandes institutions sociales. Sans qu'on observe pour autant une contestation en règle de ces institutions, on assiste à la naissance concomitante d'une multitude de regroupements ou de "microentités ». Les individus s'y retrouvent sur la base d'affinités permettant l'expression de solidarités plurielles ; en ce sens, ces regroupements « spontanés » et non mutuellement exclusifs peuvent être des tribus religieuses, sexuelles, culturelles sportives, musicales, etc. La durée de vie de ces nouvelles tribus peut être assez éphémère - en raison de leur nature même - mais chacun est libre d'en créer de nouvelles au besoin. Tout à fait parallèlement aux institutions qui cherchent à organiser une forme de cohésion sociale, on assiste donc à un processus

138 Michel Maffesoli, «De la postmédiévalité » à la postmodernité ", dans Yves Boisvert (dir.) Postmodernité et sciences humaines, Montréal, Éditions Liber, 1998, p. 9-21. 
contraire de « fragmentation de la vie sociale [...] appelée à se développer d'une manière exponentielle, constituant ainsi une nébuleuse insaisissable n'ayant ni centre précis ni périphérie discernable ${ }^{139} \%$. Cette nouvelle forme d'، être-ensemble » oscille d'ailleurs « entre l'individualisme et la masse et est davantage fondée sur l'affectif et les affinités identitaires que sur la rationalité et les identités préétablies ${ }^{140}$ ».

Quant à la troisième caractéristique de base de la postmodernité, «le bricolage mythologique », elle réfère à ce que Jean-François Lyotard a nommé « l'effritement des grands récits fondateurs ${ }^{141}$ » dont la durée de vie s'étalait souvent sur des siècles ou même des millénaires. On ne retrouve plus aujourd'hui en Occident une idéologie capable de rejoindre toutes les factions d'une société autour d'une explication, d'une interprétation ou d'une légitimation de notre histoire, de ce que nous sommes et de notre destinée. Le « localisme » et le «tribalisme » ont plutôt contribué à générer des microrécits, plus modestes, plus fugaces et facilement composites. L'humain postmoderne ne serait plus en quête d'une vérité absolue - à la manière moderne - et il aurait appris à accepter les vérités partielles, versant sans malaise dans le syncrétisme et l'éclectisme. Une autre notion peut ici nous être utile pour préciser la nature de la mutation en cours : celle d'axiologisme telle qu'utilisée par Yves Lambert ${ }^{142}$. L'axiologisme renvoie au fait que ce sont des valeurs «à la carte» - et non la référence à de quelconques dogmes ou impératifs moraux - qui constituent la forme dominante des repères de nos contemporains. L'axiologisme ainsi défini permettrait de mieux nommer cette réalité qui fait qu'on assiste à une autonomisation très poussée de ce qui guide nos actions et nos visions du monde. Nos contemporains peuvent encore partager certaines valeurs centrales en tant que repères dans leur vie, mais ces valeurs ne sont plus placées

13.) Ibid., p. 17.

1+1) Guy Ménard, "Le bricolage des dieux. Pour une lecture postmoderniste du phénomène religieux », dans Yves Boisvert (dir.) Postmodernité et sciences bumaines, op. cit., p. 111.

1+1 Cité par Guy Ménard, op. cit., p. 104.

142 Yves I ambert, Les valeurs des Frangais. Évolution de 1980 à 2000, Paris, Armand Colin, 2000. Cité par Jean-Paul Guetny, "Mort unique et deuil collectif ", Études sur la mort, n" 123, Morts et deuils collectifs, L'Esprit du temps, 2003, p. 101. 
hiérarchiquement sous la coupe d'une doctrine les chapeautant, doctrine elle-même supervisée par une institution. L'ancien ne disparait pas automatiquement du paysage, mais il n'a plus les amarres institutionnelles qui le retenaient. Les valeurs sont donc délestées des dogmes et des institutions. Elles redeviennent disponibles pour des combinaisons originales. Comme le dit Danièle Hervieu-Léger ${ }^{143}$, « le capital de références et de symboles qui appartiennent aux traditions des religions historiques ne disparait donc pas vraiment; il se trouve plutôt réinvesti, si l'on ose dire, dans de nouvelles entreprises mythicorituelles $[\ldots .]$.$» . Tout cela rend possible une grande différenciation entre$ les individus d'un même corps social, de plus en plus « déprogrammés socialement ». Comme le disent si bien les sociologues Lemieux et Montminy, «[...] le problème est moins aujourd'hui de croire que de croire ensemble $e^{144} »$.

La postmodernité ne conduit donc pas à l'aboutissement, annoncé par certains, du phénomène d'homogénéisation sociale. Au contraire, on observe en son sein un processus contraire de différenciation sociale à l'action. En effet, on remarque qu'avec la place prépondérante prise par l'individu avec l'avènement de la modernité, les relations sociales s'organisent différemment, que les influences socialisatrices sont multipliées, que la «proximité », le pluralisme, l'axiologisme et le syncrétisme marquent notre époque. Tellement que «les règles de vie en commun sont menacées ${ }^{145}$ " parce que l'individu n'accepte pas facilement les diktats venant de l'extérieur.

Toutes ces analyses informent sur les tensions qui animent les sociétés postmodernes. En effet, il apparait que, dans la postmodernité, coexistent deux mouvements antinomiques : l'un favorisant l'homogénéité, l'autre, la différence. Cette dualité de l'orientation n'est pas à nier en elle-même, car il n'y a de sociohistoire, pour l'humain, que dans la conjugaison du semblable et du dissemblable. Ce qu'il y a de particulier dans la postmodernité de cette conjugaison, c'est la pluralité et la variabilité de la

14.3 Danièle Hervieu-Léger, La religion pour mémoirt, Paris, Cerf, 1993, citée par Guy Ménard dans Yves Boisvert (dir.), op. cit., p. 107.

144 Raymond Lemieux et Jean-Paul Montminy, Le catholicisme québécois, Québec, Les Éditions de l'IQRC, I.es Presses de l'U niversité Laval, 2000, p. 107, cité par Sébastien St-Onge, L'industrie de la mort, Québec, Jes Éditions Nota bene, 2001, p. 111.

145. Simon Laflamme et Ali Reguigui, Homogénéité et distinction, Sudbury, Prise de Parole, 2003. 
différenciation et l'étendue de la tendance à l'homogénéisation. [...] Il appartient donc aux acteurs sociaux de construire, au cours des prochaines décennies, les dialectiques du semblable et du dissemblable qui leur correspondent. Mais ils doivent savoir qu'il s'agira forcément de dialectiques, donc de l'interdépendance de deux dimensions contradictoires, et que ces dialectiques ne pourront pas s'inscrire en dehors d'une société de communication de masse ${ }^{140}$.

Ce contexte général de mutation, associé à la période historique que l'on vient de décrire, ne peut être ignoré si nous espérons mieux comprendre l'évolution de nos rapports à la mort et les nouveaux visages de la ritualité funéraire.

\section{Un bilan des savoirs}

\subsection{Principales thèses des études sur la mort}

En rétrospective, nous pouvons dégager neuf thèses centrales que soutiennent les principaux auteurs dans le domaine des études sur la mort et sur les rites funéraires. Ces thèses relèvent tout à la fois de leurs observations et de leurs analyses portant, pour l'essentiel, sur les sociétés de la tradition et sur les sociétés de la modernité. Après les avoir passées en revue, nous nous demanderons si ces constats généraux valent toujours pour les sociétés de la postmodernité.

Première thèse. Il y a environ 100000 ans, avec l'invention de la sépulture, est née la culture. Si l'établissement des premières sépultures attestées est corroboré par des auteurs comme Mohen, Dortier et Vallet, d'autres auteurs comme Mauss, Morin, Thomas, Urbain, Baudry, Volant et Hulin attachent à cet événement une signification bien particulière et en font un moment fondateur de notre histoire. L'humain échappe dès lors à sa seule nature animale et accède à l'univers de la culture. Il est dorénavant habité par des questionnements nouveaux qui l'amènent à développer des pratiques rituelles funéraires qui ne répondent à aucune logique apparente de survie dans l'environnement hostile qui est le sien. Notre ancêtre prend conscience du sacré, du numineux. Il tente de mettre en scène ses origines et son destin par le biais de mythes explicatifs qui déboucheront éventuellement sur la mise au monde des

\footnotetext{
146 Simon Jaflamme et Ali Reguigui, Homogénéité et distinction, ibid., p. 223.
} 
religions primitives.

Deuxième thèse $\mathrm{e}^{147}$. Toute société se voudrait immortelle et ce qu'on appelle culture n'est rien d'autre qu'un ensemble organisé de croyances et de rites, afin de mieux lutter contre le pouvoir dissolvant de la mort individuelle et collective. Morin, Thomas, Urbain et Baudry adhèrent à cette thèse. Ils insistent ainsi sur la place centrale qu'occupent la mort et les rites dans les soubassements mêmes de l'édifice social. Leur argumentation rappelle les efforts soutenus qu'il faut consentir et les outils qu'il a fallu se donner pour continuellement faire face à la mort, individuellement et collectivement, pour réagir à sa menace et pour lui survivre. La mort dépasse notre entendement et notre raison depuis toujours. On ne peut ni la connaître, ni la contrôler, ni la maîtriser. En ce sens, elle a été une provocation fondatrice pour les liens sociaux. Il importe donc de faire remarquer que les rites funéraires sont associés de près à la naissance des sociétés humaines et aussi que ces rites ont un caractère « sauvage ", primitif, indompté, et qu'ils ont existé en dehors du cadre des religions organisées qui ne sont apparues que plus tard. On ne peut donc facilement prétendre les contrôler tout à fait non plus. D'ailleurs, pour Rivière et Ménard, les changements des dernières décennies dans la ritualité funéraire occidentale laissent croire que les rites peuvent à nouveau échapper au giron exclusif de l'institution religieuse.

Troisième thèse. La société, plus encore que l'individu, existe dans et par la mort, c'est-à-dire que la mort et les rites funéraires participent à la création sociale ou à l'institution de la société. La mort, il ne faut pas s'y tromper, n'est pas que destruction. Elle permet et elle force le renouvellement du social. Les groupements sociaux, dans toute notre histoire, ont dû s'organiser et prendre les moyens de transmettre leur patrimoine - acquis matériels, savoir-faire technique, bagage de connaissances, systèmes de croyances - par le biais d'une prise en charge d'apprentissages organisés, permettant de socialiser les membres des diverses générations d'une collectivité à un héritage commun, en lien avec un mythe fondateur. Sur le plan de la quête de sens, ils ont dû chercher également un système de mise en ordre du monde qui puisse permettre d'affronter l'outre-

I.es thèses 2, 3 et 8 de cette section sont tirées d'une synthèse des travaux de Thomas présentée par Jean-Marie Brohm dans sa préface au livre posthume de Louis-Vincent Thomas, Les chairs de la mort, op. cit., p. 25. 
cuidance de la mort au quotidien. C'est pourquoi Morin, Thomas, Brohm, Baudry, Métraux, Volant, de même que Lenoir et de Tonnac ont tous insisté, chacun à sa façon, sur le potentiel fondamental et positif de création ou de recréation sociale de la mort, lorsqu'elle est encadrée par des rites funéraires signifiants.

Quatrième thèse. Il existe quelques grands invariants dans le domaine de la mort et de la ritualité funéraire universelle, malgrél'extrême diversité et la richesse des déploiements de l'imaginaire bumain en cette matière.

Premier invariant. Les sociétés humaines, depuis l'invention de la ritualisation de la mort de l'un de leurs membres, ont en commun un même malaise en présence du cadavre d'un proche et une même horreur de la putréfaction de ce cadavre. Cela a constitué un puissant incitatif à la ritualisation et, en conséquence, elles ont mis au point trois - et seulement trois - types d'actions rituelles pour y faire face. Urbain a bien montré que l'on a ainsi pu opter, mais toujours dans une stricte logique de ritualisation, pour la destruction du cadavre (par divers moyens), pour sa dissimulation (en divers lieux) ou pour sa conservation (là aussi par diverses techniques) dans une forme jugée socialement acceptable parce qu'elle empêche justement quiconque d'être témoin de la putréfaction et parce qu'elle permet de réassigner une place au défunt. Il a aussi fait remarquer qu'il était possible de choisir une combinaison de plus d'un de ces types d'actions dans le déroulement du cycle rituel.

Deuxième invariant. Les rites funéraires sont des rites communautaires appartenant à la catégorie des rites de passage. S'il existe dans l'organisation quotidienne de la vie en société des microrites, ou rites d'interaction (Goffman), mettant en relation quelques individus seulement, les rites funéraires sont, quant à eux, de la famille des rites sociaux ou communautaires. Ils constituent également des rites de passage tels que les a définis Van Gennep et, à sa suite, beaucoup d'autres auteurs. C'est dire qu'on peut toujours les analyser et les décomposer selon les trois étapes classiques « séparation - liminarité - réintégration », ce qui correspond symboliquement, selon Dortier, à un cycle «mort - gestation - nouvelle naissance ».

Troisième invariant. Les rites funéraires remplissent universellement trois grandes fonctions sociales, d'où la nécessité de la ritualisation de la mort dans l'bistoire. 'Thomas, le premier, puis Des Aulniers et Baudry ont montré qu'au-delà de l'extrême diversité des procédés rituels, le même scénario est toujours reproduit en trois actes. Le groupe des vivants doit d'abord prendre les moyens de se séparer du cadavre après lui avoir rendu un 
dernier hommage. Ensuite, le groupe, mis à mal par la disparition de l'un des siens, prévoit une période de marge, de deuil, afin que ceux et celles qui ont côtoyé la mort et le mort de près (accompagnement, soins, toilette funéraire, etc.), et qui symboliquement du moins ont risqué une forme de contamination auprès d'une puissance qui leur échappe, puissent vivre une forme d'apaisement, de purification et de guérison avant d'être réadmis à part entière dans le groupe. Finalement, le groupe prend les moyens pour transcender la perte subie après la disparition et la mise à l'écart d'un de ses membres en la transformant en un événement positif, puisqu'on lui assigne une nouvelle place et un nouveau rôle faisant en sorte qu'il pourra continuer de participer « autrement » à la vie du groupe maintenant revivifié. Ainsi, ultimement, le rite a pour fonction de réguler la crise que le groupe traverse en permettant et en codifiant l'expression des émotions, tout en inculquant des valeurs essentielles aux membres de la collectivité au sujet du sens de la vie et de la mort.

Quatrième invariant. Les rites funéraires constituent une forme de thérapie universelle pour les vivants. Le cycle de tout rite de passage étant complété, le groupe social a pris les moyens, dans l'immédiat, de contrer le désordre faisant suite à l'irruption de la mort et, à plus long terme, il a aussi pris les moyens de nier à la mort son pouvoir dissolvant et annihilant qui serait sinon un échec sans retour pour l'individu et le groupe. Par conséquent, le message symbolique à l'issue des rites funéraires est clair : la vie est plus forte que la mort. En ce sens, Thomas, Des Aulniers et Baudry insistent tous sur le caractère universellement thérapeutique des rites funéraires, autant pour l'individu endeuillé que pour l'inconscient collectif.

Cinquième invariant. Les rites funéraires constituent depuis toujours un système organisé de déni symbolique de la mort. Refusant, il y a 100000 ans, que la mort soit le signe d'un anéantissement final, nos ancêtres ont imaginé, grâce à ce que nous avons appelé une ruse de la pensée, que seul notre corps était anéanti avec la mort. Subsiste donc l'autre partie que l'on nomme âme, esprit ou double en fonction des mythes fondateurs. C'est pourquoi Urbain a défini très brièvement mais très justement, nous semble-t-il, les rites funéraires comme étant « une imagination organisatrice de la disparition ». Thomas et Baudry abondent dans le même sens en rappelant que les rites ne sont pas là pour apprivoiser la mort, qu'aucune société ne l'a apprivoisée, mais pour soustraire les 
membres du corps social à la violence sans nom de cette rencontre répétée avec la mort. Ariès avait pour sa part fait une autre utilisation de l'expression «déni de la mort » en prétendant que notre époque était la première à avoir ce rapport pathologique avec la mort. Pour Thomas et Baudry, par contre, le déni symbolique de la mort est toujours, au contraire, une réaction saine des sociétés, la seule possible et pensable. La caractéristique problématique des sociétés occidentales modernes, c'est d'avoir développé un réflexe de «déni réel » devant la mort, qui se résume essentiellement à l'exclusion et à l'oubli de la réalité de la mort du cour de la vie individuelle et sociale en la refoulant au terme de l'existence, en la limitant à la terminaison de la vie d'un individu, comme si elle n'avait pas un impact individuel et collectif subversif tout au long de notre vie. Il faut bien voir qu'en excluant la mort de nos vies, on ne peut plus se prémunir individuellement ni collectivement contre ses effets et que vouloir l'oublier ou la nier, c'est en fait lui donner toute la place et lui redonner tout son potentiel de violence symbolique presque imparable sans médiation rituelle. En ce sens, la modernité occidentale nous aurait fait développer un nouveau rapport à la mort de nature pathologique.

Sixième invariant. Il existe dans notre bistoire un nombre limité de visions ou d'idéologies eschatologiques qui contribuent à définir autant de rapports à la mort de nature différente. Plusieurs auteurs font des classifications quelque peu différentes des eschatologies existantes. Morin insiste sur le fait que les deux sources originelles des eschatologies s'appuient soit sur l'idée de renaissance, soit sur celle de survie. Thomas distingue quatre possibilités allant de la néantisation totale lors du décès, à la renaissance ou métamorphose, à l'idée de survie indirecte par des traces que l'on laisse de notre passage et à l'amortalité que procurera un jour la science à l'espèce. DeSpelder et Strickland, de même que Balandier arrivent à des conclusions similaires, sous une forme binaire : ou bien notre destin mène à un seuil ou à un passage qui permet l'espérance et la projection vers un ailleurs, ou bien il aboutit à un cul-de-sac, à un mur, au néant, à la disparition pure et simple. Balandier laisse aussi entrevoir le développement d'une conception poussant l'espérance jusqu'à compter sur l'avènement de l'amortalité. Quant à Gaudin, il décrit les modèles possibles en fonction des formes de vie qui sont réservées au défunt: une forme de survie où les humains ne sont que de pâles reproductions de leur vivant; une forme de survie où tout est amplifié, en bien ou en 
mal ; une existence terrestre invisible dans le village des morts ; une forme de survie où seule une partie de l'individu subsiste ; un anéantissement de la personne jusqu'à ce qu'une intervention extérieure lui redonne vie. On remarque que Morin et Gaudin sont les seuls à ne pas inclure, dans leur classification respective, la possibilité qu'il n'y ait pas de survie.

Septième invariant. On observe universellement l'existence et le développement d'une importante culture funéraire matérielle dans l'bistoire, depuis l'apparition des premières sépultures. Toutes les cultures ont, selon les auteurs consultés, pris soin de conserver des traces, d'une manière ou d'une autre, de leurs morts. Il ne suffit pas de ritualiser le moment du passage pour assurer le devenir eschatologique de nos proches. Encore faut-il leur trouver une place, les associer à des objets dans une logique de conservation de traces de leur passage sur terre. Morin, Thomas, Urbain, Mohen, Javeau, de même que Lenoir et de Tonnac en arrivent tous au même constat. Urbain, Thomas, Lenoir et de Tonnac notent, par contre, qu'avec le développement progressif de la crémation en Occident à partir des années soixante, combiné à la pratique de la dispersion des cendres, ce vieil invariant est peut-être remis en cause pour la première fois. Urbain décèle, quant à lui, dans les pratiques crématoires récentes, une modification de nos habitudes sans les assimiler à une rupture totale de la volonté de conservation des traces des défunts. Il les associe plutôt à une privatisation du culte des morts.

Cinquième thèse. Les rites funéraires constituent un espace et un système de communication d'un type bien particulier. En se basant sur les travaux de Lardellier, on constate que les rites funéraires, en tant que rites de passage, permettent une forme de communication sociale de nature formelle avant tout. Toutefois, en regroupant justement un grand nombre de participants dans un contexte bien particulier - où la communication sociale peut devenir communion sociale étant donné l'atmosphère générale de quête de sens qui y règne -, on assiste souvent à une intensification des relations sociales pendant ce que Lardellier appelle la «liminarité rituelle », c'est-à-dire la période où l'on prépare le rite, où on le vit et qui peut même se poursuivre un temps dans l'après-rite. Cette intensification des relations sociales favorise le développement de liens sociaux temporaires, hors hiérarchie, dans un contexte d'« anti-structure sociale ", comme le disait Turner, mais des liens sociaux potentiellement très intenses, ce qui est rare en situation de communication sociale 
formelle. C'est pourquoi Lardellier propose la notion de « creuset rituel », parce que le rite provoque une accélération des relations sociales entre des gens potentiellement très différents selon les catégories sociologiques classiques, mais qui sont réunis pour la même chose autour du rite. Ajoutons que l'impact de cette communication rituelle repose sur une combinaison de dispositifs de nature technique tout autant que de nature symbolique, tout en profitant d'un rapport au temps différent de ce qu'il est lorsque chacun vaque à ses autres occupations. De plus, la communication rituelle ne se limite pas à la communication qui s'instaure entre les participants au rite, mais elle s'installe aussi entre les participants et des mythes et des croyances.

Sixième thèse. En raison de l'utilisation intensive qui est faite du symbolisme dans la pratique rituelle et dans la ritualité funéraire en particulier, les participants "volontaires » à un rituel réussi expérimenteront un changement de registre mental et de cadre social. Morin, Thomas, Des Aulniers, Lardellier, Jeffrey, Gruau et Urbain ont tous insisté sur l'importance de l'imaginaire et des symboles collectifs, issus des mythes fondateurs, pour affronter ce qui dépasse ou menace l'individu. Comme il refuse l'enfermement où le conduit la mort des siens, le recours aux symboles et à la médiation rituelle lui permettra de donner un sens à ce qui n'en n'a pas. Comme l'ont montré Mauss et Lardellier, par le déploiement et la médiation de la symbolique rituelle, les participants sont amenés à voir et à comprendre les choses sous un autre angle, en sortant de l'ordre normal et habituel des choses. Dans ce contexte, le langage symbolique vient secourir la logique rationnelle chancelante et inopérante. Le recours aux symboles amène à réinterpréter la mort avec et selon un nouveau code et grâce à ce contexte qui oblige à quitter le cadre social habituel où certaines choses ne sont pas possibles, tout en étant nécessaires.

Septième thèse. Les rites funéraires comportent deux dimensions sociologiques : l'individuation et la socialisation. C'est Javeau qui explique que les rites funéraires sont l'occasion de produire deux discours et de poser des gestes de deux ordres : on rend hommage au défunt et on reconnaît son histoire et sa singularité dans un monde où les individus constituent les atomes du grand jeu social ; mais on se charge aussitôt de le réintégrer dans le circuit social, dans le groupe des bâtisseurs et des ancêtres qui devient pour les vivants une référence et un guide.

Huitième thèse. La mort, du moins l'usage social qui en est fait, constitue un des grands révélateurs des sociétés et des civilisations, donc de leur questionnement 
et de leur critique. Thomas et à sa suite Baudry ont mis l'accent sur le fait que les études sur la mort et sur nos rapports à la mort et aux rites ne sont pas des objets de recherche comme les autres, mais qu'ils concernent une réalité fondatrice de nos organisations sociales et de notre histoire. Cet objet d'étude pluriel constitue en lui-même un paradigme socioanthropologique fondamental, puisque l'être social se construit dans sa résistance au néant et qu'en ce sens la mort est toujours relation à autrui, mort ou vivant. Ce paradigme thanatologique sert d'outil à l'analyse, à la compréhension et même à la critique de nos formations sociales.

Neuvième thèse. Les rites funéraires se transforment avec les grands changements sociaux qui traversent l'bistoire des sociétés humaines. Que la forme des rites funéraires change avec le temps dans l'histoire, voilà un fait bien établi à la suite des travaux de pionniers comme Morin, Ariès, Thomas, Vovelle et Ziegler. Si le passage des sociétés de la tradition à celles de la modernité a révolutionné nos modes d'être ensemble, cela n'a pas été sans effet sur notre vision de la vie et de la mort. De la même façon, le passage ou la mutation qui s'opère entre modernité et postmodernité, dans l'ensemble de l'organisation sociale, produit donc aussi ses effets sur nos pratiques funéraires contemporaines ${ }^{148}$. Louis-Vincent Thomas a beaucoup écrit sur le sort des rites funéraires au cours des dernières décennies. Selon lui, si on a pu reconnaitre dans les sociétés de la

14x Les Michel Maffesoli [" De la "postmédiévalité " à la postmodernité ", dans Yves Boisvert (dir.), Postmodernité et sciences bumaines, Montréal, Éditions Liber, 1998], Yves Boisvert « Sortir du nihilisme. Quand la dictature du moi sert de bouée», dans Yves Boisvert et Lawrence Olivier (dir.), A chacun sa quête. Essais sur les noweaux visages de la transcendance, Sainte-Foy, Presses de l'Université du Québec, 2000 et "L'analyse postmoderniste au-delà de l'esthétisme discursif ", dans Yves Boisvert (dir.), Postmodernité et sciences bumaines, Montréal, Éditions Liber, 1998], Georges Balandier [ D'une espérance à l'autre. L'émergence de l'homme amortel », dans Frédéric Lenoir et Jcan-Philippe de Tonnac (dir.), op. cit.], Gilles Lipovetsky [L'ère du vide, Paris, Gallimard, 1983], Jean-François Lyotard [cité par Guy Ménard, Petit traité de la vraie religion à l'usage de ceux et celles qui soubaitent comprendre un peu mieux le vingt et unieme siècle, Montréal, Liber, 1999], Charles Taylor [I a diversité de l'expérience religieuse aujourd'bui, Montréal, Bellarmin, 2003], Denis Jeffrey (" Éthique et postmodernité », op. cit.], ainsi que Simon Laflamme et Ali Reguigui [Homogénéité et distinction, op. cit.], en cherchant à dépeindre en quoi consiste cette transition en action dans nos sociétés, nous offrent une grille générale pour décoder et pour comprendre les analyses et les interprétations de Thomas. 
tradition l'importance centrale de la ritualité, on a pu observer une tendance à l'obsolescence progressive de nos rites funéraires associés à la modernité, alors qu'avec la postmodernité, on assisterait à un début de renaissance relative de la ritualité funéraire. Dans quelle mesure les neuf thèses dégagées des travaux de la thanatologie au sens large continuent de s'appliquer au monde de la postmodernité ? C'est ce que nous explorons dans la prochaine section.

\subsection{Questionnement critique}

À ce stade de la recherche, il est possible de faire le point sur les possibilités qui s'ouvrent à de nouvelles enquêtes sur la ritualité funéraire et sur nos rapports contemporains à la mort en Occident. Nous prendrons appui sur le bilan de notre recension pour faire ressortir les nouvelles questions qui se posent à partir des analyses de l'ensemble des auteurs dont nous avons voulu résumer les thèses principales. Ces nouvelles questions de recherche seront liées à la ritualité funéraire en contexte postmoderne.

Parmi les neuf thèses présentées dans la section précédente, trois nous semblent assez bien établies et ne pas soulever de nouvelles interrogations importantes malgré les mutations sociales induites avec le passage progressif à la postmodernité. Dans le cas des six autres, nous souhaitons vérifier si les constats établis par les auteurs à partir de l'observation et de l'étude des sociétés traditionnelles et modernes sont toujours valables avec les mutations caractéristiques de la postmodernité.

Première thèse. Ily a environ 100000 ans, avec l'invention de la sépulture, est née la culture. Cette thèse ne relève pas des changements sociohistoriques qui ont pu survenir dans nos sociétés depuis la naissance de la culture. Cette idée semble suffisamment plausible pour ne pas la remettre en question, avec toutefois le bémol suivant : dans les écrits scientifiques, le débat fait toujours rage à propos de ce qui a pu être le facteur décisif permettant l'émergence de la culture ${ }^{149}$. Mais le rôle qu'y

14" Voir à ce propos le récent dossier spécial qu'y a consacré la revue Sciences bumaintes dans son premier numéro d'une nouvelle parution trimestrielle : Jean-François Dortier (dir.), "L'origine des cultures", dans Les grands dossiers des sciences bumaines, $\mathbf{n}^{\circ} 1$, décembre 2005/janvier 2006, p. 26-94. 
ont probablement joué la prise de conscience de notre finitude et le développement des rites funéraires qui ont suivi assurent à cette thèse une crédibilité suffisante pour qu'on lui fasse au moins une place parmi les facteurs déclencheurs de la naissance de la culture.

Deuxième thèse. Toute société se voudrait immortelle et ce qu'on appelle culture n'est rien d'autre qu'un ensemble organisé de croyances et de rites, afin de mieux lutter contre le pouvoir dissolvant de la mort individuelle et collective. Cette thèse constitue une suite logique de la première. Même si elle semble vouloir réduire la culture à un outil pour affronter la mort, cela ne nous empêche en rien de continuer de voir dans la culture ce que la sociologie classique lui a reconnu comme rôle. La formulation actuelle de cette thèse a, par contre, l'avantage de reconnaitre le lien étroit entre la naissance de la culture et la prise de conscience de la mort. C'est pourquoi nous ne la contestons pas ici. Rien n'indique d'ailleurs que l'entrée dans la postmodernité change quoi que ce soit à ce constat, mais une meilleure connaissance des effets de la culture postmoderne sur les rites funéraires pourrait nous amener à revoir notre position à ce sujet. En effet, est-il possible qu'avec la culture postmoderne on assiste à un déplacement de nos croyances qui, auparavant, allaient dans le sens du refus du pouvoir destructeur de la mort en imaginant un "après », et qui, maintenant, s'organiseraient autour du refus de la mort comme destin inéluctable de l'individu qui, aidé de la biomédecine, pourrait devenir amortel ? Si tel était le cas, c'est tout de même encore l'univers de la culture et des croyances qui s'outille autrement pour affronter le même pouvoir dissolvant de la mort. En ce sens, la thèse reste valable malgré l'entrée en scène de la postmodernité.

Troisième thèse. La société, plus encore que l'individu, existe dans et par la mort, c'est-à-dire que la mort et les rites funéraires participent à la création sociale ou à l'institution de la société. Nous comprenons bien l'idée selon laquelle la mort et les rites funéraires participent à la création ou à l'institution de la société. Toutefois, il faut se demander si cette valence positive de la mort peut être maintenue dans un contexte social qui serait marqué par une forme de « déni réel » de la mort en Occident, cette forme de déni ayant commencé à s'exprimer dans le cadre de la modernité. En effet, comment la mort et les rites pourraient-ils conserver intact ce pouvoir de création ou de recréation sociale lorsque la place même de la mort est niée par un nombre important de nos contemporains qui n'acceptent la réalité de la mort que comme terminaison de la vie (et encore pas tous, 
puisque le rêve de l'amortalité se fait séduisant pour un certain nombre d'entre eux) et refusent d'y voir autre chose, en la considérant simplement comme la disparition d'une personne, et rien de plus. Ce pouvoir de création sociale est-il alors affaibli ou même nié puisqu'on ne déploie plus la même énergie qu'avant à rejeter symboliquement et socialement cette réalité de la mort qu'on traite plutôt comme une disparition sans lendemain ? En ce sens, on peut formuler la proposition suivante : avec la postmodernité, l'expérience de la mort d'un membre de la société ne permet pas, ni pour la collectivité ni pour les proches, la pleine expression du pouvoir de recréation sociale de la mort, tel que relevé dans les écrits pour les périodes précédentes (et cela nous semble d'autant plus probable lorsque les rites font dans le minimalisme).

En outre, les rites funéraires actuels sont-ils encore en mesure de transmettre des apprentissages et de socialiser les membres des diverses générations à un héritage social commun en contexte de face à face avec la mort? La question se pose puisque, d'une part, moins de gens sont rassemblés pour l'occasion, et que, d'autre part, les récits fondateurs collectifs ont éclaté en une kyrielle de micromythes. Cette deuxième question permet de poser une seconde proposition : avec la postmodemité, les «nouveaux rites » funéraires - lorsqu'ils existent - n'ont plus la capacité de favoriser des apprentissages permettant la transmission d'un mode de réaction et d'un béritage social appartenant à un cadre social collectivement partagé.

Quatrième thèse. Il existe quelques grands invariants dans le domaine de la mort et de la ritualité funéraire universelle, malgré l'extrême diversité et la richesse des déploiements de l'imaginaire bumain en cette matière.

Premier invariant. Les sociétés bumaines, depuis l'invention de la ritualisation de la mort de l'un de leurs membres, ont en commun un même malaise en présence du cadavre d'un proche et une même borreur de la putréfaction de ce cadavre. Cela a constitué un puissant incitatif à la ritualisation et, en conséquence, elles ont mis au point trois - et seulement trois - types d'actions rituelles pour y faire face. Ici, la question que nous souhaitons soulever ne remet pas en cause l'existence de trois modes de disposition du corps. Seulement, avec la combinaison du développement des arrangements funéraires préalables, de la popularité grandissante de la crémation et de l'abandon de certaines pratiques funéraires, c'est plutôt la nécessité même de la ritualisation des modes de disposition du corps qui semble remise en cause. En effet, c'est surtout par l'intermédiaire de la volonté préalablement exprimée du défunt ou encore sous l'effet de l'adoption grandissante de la technique de crémation que l'absence de ritualisation d'un moment important des 
gestes entourant le décès d'une personne est observé de plus en plus fréquemment aujourd'hui. Lorsque les dernières volontés d'un défunt vont dans le sens de ne pas trop "déranger", de ne pas imposer des moments jugés trop difficiles aux survivants - entre autres, en optant pour l'incinération qui ne prévoit ni la participation ni la présence des proches, et constitue un acte purement technique - , la ritualisation apparaît en pareil cas comme amputée d'un premier geste très significatif pour les sociétés qui nous ont précédés, et ce, même si une cérémonie publique est prévue autour de cet événement, soit avant, soit après. On peut, en conséquence, se demander si la ritualisation qui, historiquement, selon Urbain, accompagne les gestes entourant la disposition d'un cadavre est toujours ressentie comme nécessaire aujourd'hui ? Sous forme de proposition : avec la postmodernité, le réflexe de ritualiser le moment de la disposition du corps mort s'estompe.

Deuxième invariant. Les rites funéraires sont des rites communautaires appartenant à la catégorie des rites de passage. Premièrement, doit-on encore utiliser le concept de rites communautaires ou sociaux quand on observe les faibles assistances, surtout en milieu urbain, au moment de la tenue des rituels, si toutefois ceux-ci existent et sont publics, ce qui n'est plus toujours un automatisme aujourd'hui. Les « nouveaux rites » observés regrouperaient un nombre plus restreint de participants, réunis pour une plus brève période, dans un lieu qui n'a pas nécessairement affaire avec le culte. En ce sens, devrait-on plutôt parler de « rites de proximité ${ }^{150}$ »? Notre proposition prendrait en conséquence la forme

1.31 Concept emprunté à Fabricc Hervicu-Wane, Une boussole pour la vie. Les nouveaux rites de passage, Paris, Albin Michel, 2005, p. 97-98. Cet auteur utilise aussi les notions de "rites sur mesure » et de "rites à la carte " pour parler des nouveaux rites de passage qu'elle observe dans la société contemporaine. Luce Des $\Lambda$ ulniers avait quant à elle utilisé les notions de "rites intimes", de " microrituels", de «rites privés", «mésocollectifs» ou «macrocollectifs»dans le numéro spécial de la revue Frontières à l'occasion du $10^{\mathrm{c}}$ anniversaire de cette publication en 1998. Hervieu-Wane donne toutefois une connotation péjorative à sa notion de rite de proximité, lui reprochant un manque de reconnaissance sociale à partir du postulat que les rites ne valent que s'ils sont partagés par tous. De notre côté, nous souhaitons conserver une coloration neutre à la notion, en ne présupposant rien de leur impact social à ce stade-ci. Nous avons également retenu cette appellation sous l'influence de Michel Maffesoli qui a insisté, dans sa description des caractéristiques de la postmodernité, sur l'importance des liens de proximité et des valeurs de proximité. 
suivante : avec la postmodemité, les rites funéraires qui subsistent se transforment en rites de proximité.

Deuxièmement, peut-on encore parler de rites de passage puisque les cérémonies funéraires actuelles ne sont souvent plus l'occasion, à la suite de la disparition des grands mythes fondateurs socialement partagés, de réfléchir systématiquement sur le destin eschatologique du défunt, mais se limitent souvent à lui rendre hommage en rappelant qui il a été pour ses proches et ce qu'il a accompli. Le devenir eschatologique est ainsi devenu une question de croyance personnelle plutôt qu'une croyance religieuse socialement définie et transmise. Il s'agit d'un changement qui n'est pas sans conséquences, puisqu'un rite de passage doit permettre la réintégration sociale de la personne concernée à la suite de la redéfinition de son changement de statut. À ce sujet, notre proposition irait maintenant dans le sens suivant : avec la postmodernité, les rites funéraires s'éloignent de la logique des rites de passage en renonçant à aborder la question de l'avenir post-mortem.

Troisième invariant. Les rites funéraires remplissent universellement trois grandes fonctions sociales, d'où la nécessité de la ritualisation de la mort dans l'bistoire. Deux questions se posent à notre avis au sujet des fonctions sociales universelles des rites. Pour commencer, ce qu'on appelle les " nouveaux rites » ou le phénomène de la personnalisation des rites ou ce que nous avons proposé de nommer des rites de proximité ont-ils la capacité d'assumer les trois fonctions sociales usuelles des rites funéraires selon 'Thomas? Cette première question soulève trois propositions distinctes : avec la postmodernité, les rites funéraires s'occupent toujours de la gestion du corps mort - remplissant ainsi la première des deux exigences de la première fonction sociale de tout rite funéraire-, mais la seconde exigence-consistant à prendre en charge le devenir eschatologique du défunt-, est le plus souvent ignorée.

Concernant, la seconde fonction dite d'apaisement des endeuillés, les pratiques contemporaines passent souvent dorénavant par une forme de professionnalisation ou de médicalisation du deuil, faisant ici référence soit à la prescription de médicaments pour « endormir » la douleur associée à la perte, soit à la pratique de référer l'endeuillé le plus tôt possible à un thérapeute ou à un groupe de soutien pour les personnes endeuillées. Tout cela donne l'impression que les rites parviennent moins à assurer ce rôle d'apaisement, ce qui nous amène à formuler cette proposition : avec la postmodemité, les rites funérairés n'assument que partiellement la deuxième fonction - consistant à assurer l'apaisement pour 
l'endeuillé -, dès lors que les rites de deuil sont à toute fin pratique disparus du paysage des rites contemporains.

Quant à la troisième fonction des rites funéraires, celle qui interpelle leur capacité à ressouder la collectivité en donnant un sens à un événement aussi menaçant que la mort de l'un de ses membres, l'ancien caractère public de l'événement se dissout de plus en plus et on pourrait parler plutôt de la quasi-invisibilité sociale des rites de proximité, d'où la proposition qui suit : avec la postmodernité, les rites funéraires ne concernent plus, le plus souvent, la collectivité, mais des microgroupes réunis autour de la connaissance immédiate du défunt.

La deuxième question qui se pose au sujet des trois fonctions traditionnelles des rites funéraires est la suivante : est-il possible que ces fonctions soient toujours là, bien vivantes, mais qu'elles soient assurées d'une autre manière, prises en charge par une autre instance sociale, par un autre médium que les rites? Si c'est le cas, on évaluerait autrement la prétendue déliquescence des rites funéraires amorcée avec la modernité. En effet, dans un contexte où notre rapport à la mort s'est considérablement transformé, de même que notre rapport au social, l'entreprise de mise en sens du monde ne constitue plus un apprentissage collectif et l'expérience du face à face avec la mort n'étant plus vécue dans le registre du collectif, il faut chercher ailleurs que dans les seuls rites communautaires, par définition essentiellement sociaux, la nouvelle façon plus individuelle de répondre à ce qu'on a appelé la provocation de la mort. Notre proposition serait la suivante : avec la postmodernité, les grandes fonctions usuelles assumées par les rites funéraires jusqu'à la modernité ne sont plus l'apanage des seuls rites.

Quatrième invariant. Les rites funéraires constituent une forme de thérapie universelle pour les vivants. Pour que les rites aient une action thérapeutique, il faut, nous semble-t-il, que l'on prenne acte réellement de la présence de la mort et pas seulement au terme de la vie. Il faut aussi faire face aux questionnements de nature eschatologique. S'il est vrai, comme le prétendent, entre autres, Thomas, Des Aulniers et Baudry, que notre société occidentale ait développé une forme de "déni réel » de la mort à notre époque, la "magie thérapeutique » peut-elle toujours opérer, aussi bien sur le plan individuel que social ? Si, malgré la présence des rites, on continue à être obnubilés par ce qui arrive à nos proches après la mort et, par extension, par ce qui attend chaque personne, le caractère conjuratoire avéré de l'opération semble pour le moins incertain. Notre 
proposition prendrait alors la forme suivante : avec la postmodernité, les rites funéraires ont un moindre impact thérapeutique.

Cinquième invariant. Les rites funéraires constituent depuis toujours un système organisé de déni symbolique de la mort. Qu'en est-il des rites funéraires de proximité ? Jouent-ils toujours à cet égard le même rôle ou participent-ils plutôt au déni réel de la mort comme Thomas et Baudry l'ont dit à propos de notre époque et de notre civilisation? Si c'était le cas, ils parviendraient plus difficilement à nier ultimement à la mort son pouvoir dissolvant pour l'individu et pour la société. Ce questionnement repris sous forme de proposition donnerait ce qui suit à la place : avec la postmodernité, les rites funéraires ont tendance à se limiter à constater le décès ou la disparition d'un individu et à lui rendre un dernier bommage.

Sixième invariant. Il existe dans notre histoire un nombre limité de visions eschatologiques qui contribuent à définir autant de rapports à la mort de nature différente. Puisque la postmodernité serait caractérisée par une forme de réenchantement du monde, d'une part, et aussi, d'autre part, par le bricolage mythologique, donnant naissance à des microrécits, l'éventail des possibles sur le plan des idéologies eschatologiques s'en trouve-t-il modifié ? Comment nos contemporains se situent-ils sur le continuum qui fait de la mort un seuil à franchir, à une extrémité de ce continuum, et, à l'autre, un mur infranchissable où l'on se brise inexorablement. En fait, ne devrait-on pas ajouter deux autres possibilités - hors continuum - qui consisteraient à prétendre que la mort n'appartient pas nécessairement au destin de l'humain, qu'elle pourra bientôt être évitée avec l'amortalité (Thomas et Balandier) ou encore que l'on ne disparaît jamais complètement (Thomas)?

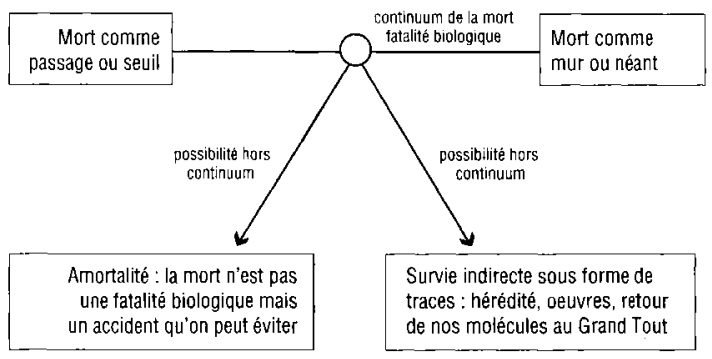


Des changements de cette nature sont majeurs et vont dans le sens des thèses sur la diversification de la culture - dans ce cas-ci funéraire plutôt que de participer à un courant d'homogénéisation culturelle. De plus, on a dit plus haut que les visions eschatologiques sont reliées de près aux rites funéraires qu'une culture met sur pied et qu'elles conditionnent notre rapport à la mort. On peut par conséquent en tirer les deux propositions suivantes : avec la postmodemité, les croyances ou idéologies eschatologiques se diversifient et se multiplient; et avec la postmodernité, la diversification des idéologies eschatologiques contribue au développement des rites de proximité.

Septième invariant. On observe universellement l'existence et le développement d'une importante culture funéraire matérielle dans l'bistoire, depuis l'apparition des premières sépultures. Urbain, Thomas, Lenoir et de Tonnac ont déjà mis le doigt sur une possible dérogation contemporaine à cet invariant en faisant remarquer que le développement rapide de la crémation dans plusieurs pays occidentaux, en lien avec l'usage que l'on fait ensuite des cendres, ainsi que les modes de développement des cimetières urbains qui auraient tendance à s'effacer du paysage ou à s'y fondre, tout cela remet en question la tendance générale observée jusque-là. Il faudrait donc voir de plus près et tenter de mesurer ce phénomène avec plus de précision. Il faudrait de la même manière prêter attention aux nouvelles formes prises éventuellement par cette culture funéraire matérielle, que ce soit dans le registre du privé (comme avec le montage des histoires de vie conservées sur support vidéo) ou dans celui du social (comme avec les cimetières virtuels dans Internet) ou encore dans un registre davantage symbolique que matériel (comme lorsque la dispersion des cendres du défunt est perçue comme un retour à la nature ou comme une fusion dans le Grand Tout cosmique). Demandons-nous si notre époque ressent encore ce besoin de participer à une culture matérielle axée sur la préservation de traces de nos morts par le biais d'objets ou de lieux. La proposition qui découle ici de notre réflexion se lit comme suit : avec la postmodernité, certains modes traditionnels de conservation des traces de nos morts s'estompent, alors que d'autres s'inventent.

Cinquième thèse. Les rites funéraires constituent un espace et un système de communication d'un type bien particulier. Est-ce que les «nouveaux rites» parviennent toujours, par une médiation à la fois technique et symbolique, à provoquer une intensification des relations sociales en lien avec la liminarité rituelle (Lardellier) et à obtenir des « résultats » malgré la 
réduction du nombre des participants? Si, dans le cadre de la mise en scène des rites de proximité, les symboles utilisés, comme les mythes sur lesquels ils reposent, ne constituent plus une référence collective, quel genre de liens sociaux s'y nouent et entre qui ? Notre proposition serait que : avec la postmodernité, les rites funéraires parviennent plus difficilement à provoquer un effet d'intensification des relations sociales.

Sixième thèse. En raison de l'utilisation intensive qui est faite du symbolisme dans la pratique rituelle et dans la ritualité funéraire en particulier, les participants "volontaires » à un rituel réussi expérimenteront un changement de registre mental et de cadre social. Avec la fin des grands récits mythiques largement partagés dans une population et le passage à ce qu'on a appelé le bricolage mythologique, qu'advient-il des symboles utilisés à l'occasion des « nouveaux rites " funéraires ? Quelle est leur nature et quels sont leurs liens avec le social ? Parviennent-ils encore à susciter un changement de cadre social et d'état d'esprit chez les participants même lorsque ces derniers " démissionnent » devant le temps requis pour véritablement entrer dans le rite ? Sous forme de proposition, nous pourrions avancer que : avec la postmodernité, les rites funéraires et les symboles les accompagnant arrivent peu à provoquer un changement de registre mental ou une transformation du cadre social des participants au rite.

Septième thèse. Les rites funéraires comportent deux dimensions sociologiques: lindividuation et la socialisation. Est-il toujours juste de dire avec Javeau que ces deux dimensions sont présentes aujourd'hui ? Les « nouveaux rites » permettent-ils aux défunts de s'inscrire dans une lignée tout en s'individualisant ou ont-ils pour résultat d'individualiser chaque histoire en omettant la composante de socialisation ou d'inscription dans une lignée, ce qui était typique du rite funéraire traditionnel ? Formulons ici la proposition qui suit : avec la postmodemité, les rites funéraires insistent sur la composante "individuation» et sur l'bistoire personnelle du défunt, tout en mettant en sourdine la composante « socialisation ».

Huitième thèse. La mort, du moins l'usage social qui en est fait, constitue un des grands révélateurs des sociétés et des civilisations, donc de leur questionnement et de leur critique. Les études et travaux des diverses disciplines des sciences humaines sur la mort et le social depuis la publication du livre de Morin en 1951 convergent dans cette direction. On a donc vu juste, même si beaucoup reste à faire pour exploiter au maximum cette intuition et les premières recherches qu'elle a produites. C'est spécialement vrai dans le contexte du développement d'une civilisation postmoderne dont il 
faut encore saisir toute la portée alors que nous y baignons : position difficile pour les chercheurs...

Neuvième thèse. Les rites funéraires se transforment avec les grands changements sociaux qui traversent l'bistoire des societés bumaines. Une fois ce constat établi, il faut maintenant examiner de plus près spécialement les mutations rituelles à l'œuvre avec l'entrée dans le postmodernisme. Estil possible que les rites funéraires aient été l'outil privilégié des sociétés traditionnelles pour faire face à la mort, outil que la modernité aurait délaissé ? La postmodernité, quant à elle, réglerait-elle ses comptes avec la mort autrement? Assiste-t-on à une véritable renaissance des rites funéraires? L'expression maintes fois utilisée de «nouveaux rites » estelle justifiée? Si oui, en quel sens? Par ailleurs, puisque les rites sont rattachés à un contexte social connu, observe-t-on une tendance à l'homogénéisation des rites ou à leur hétérogénéisation ? C'est l'ensemble des questions et hypothèses posées concernant les autres thèses qui nous permettra de mieux répondre à ces questions.

\section{Synthèse sous forme d'hypothèses}

Si les sociétés de la tradition ont presque 100000 ans d'histoire de face à face avec la mort, les sociétés de la modernité en ont à peine deux siècles et celles de la postmodernité ne peuvent revendiquer que quelques décennies. Les conclusions auxquelles les chercheurs sont arrivés sur les premières sont de mieux en mieux établies et connues. Quant aux sociétés modernes, malgré leur courte histoire, elles ont été beaucoup étudiées, notamment parce que leur apparition correspond, en gros, à celles de plusieurs sciences humaines. Pour ce qui est de la période postmoderne, son fondement heuristique, bien que jugé prometteur par de nombreux intellectuels, ne fait pas encore l'unanimité. Dans le cadre des études sur la mort et les rites, beaucoup reste à faire à ce sujet. Nous avons bien en main quelques observations et des questions savantes la concernant, mais leur nature souvent spéculative trahit soit le faible degré d'avancement de nos sciences concernant cette période, soit la rareté des enquêtes empiriques à son sujet, soit la nécessité de renouveler notre regard en fonction d'un objet nouveau qui ne peut être appréhendé de façon satisfaisante avec les grilles développées surtout dans et pour la période précédente. C'est pourquoi nous avons fait remarquer que les grands constats scientifiques dont nous 
disposons dans le champ des connaissances sur la mort et les rites sont issus principalement de l'observation et de l'analyse des sociétés traditionnelles et des sociétés modernes.

En conséquence, le questionnement devient le suivant : comment les rites funéraires de l'Occident postmoderne se situent-ils par rapport aux façons de « faire du rite » développées par nos ancêtres et prédécesseurs jusqu'à l'époque moderne telles que la recherche les a révélées? Doit-on parler de continuité, d'adaptation, de disparition, de déplacement, de renouvellement, de rupture, d'amalgame ou d'invention?

Nous avons déjà soulevé un certain nombre de propositions quant à la façon dont les rites funéraires évoluent actuellement en Occident postmoderne. Rappelons-les ici.

- Avec la postmodernité, l'expérience de la mort d'un membre de la société ne permet pas, ni pour la collectivité ni pour les proches, la pleine expression du pouvoir de recréation sociale de la mort, tel que relevé dans la littérature pour les périodes précédentes. $[\text { Thèse } 3]^{151}$

- Avec la postmodernité, les «nouveaux rites » funéraires - lorsqu'ils existent - n'ont plus la capacité de favoriser des apprentissages permettant la transmission d'un mode de réaction et d'un héritage social appartenant à un cadre social collectivement partagé. [Thèse 3]

- Avec la postmodernité, le réflexe de ritualiser le moment de la disposition du corps mort s'estompe. [Thèse 4, invariant 1]

- Avec la postmodernité, les rites funéraires qui subsistent se transforment en rites de proximité. [Thèse 4, invariant 2]

- Avec la postmodernité, les rites funéraires s'éloignent de la logique des rites de passage en renonçant à aborder la question de l'avenir post-mortem. [Thèse 4 , invariant 2]

- Avec la postmodernité, les rites funéraires s'occupent toujours de la gestion du corps mort - remplissant ainsi la première des deux exigences de la première fonction sociale de tout rite funéraire - mais la seconde exigence - consistant à prendre en charge le

15. Nous indiquons ici, à l'intéricur de l'accolade, la numérotation de la thèse de référence (en lien avec la partic 3.1) ayant servi à mettre en lumière chacune des propositions découlant des nouvelles questions de recherche qui nous sont apparues. 
devenir eschatologique du défunt - est le plus souvent ignorée. [Thèse 4, invariant 3]

- Avec la postmodernité, les rites funéraires n'assument que partiellement la deuxième fonction - consistant à assurer l'apaisement pour l'endeuillé -, dès lors que les rites de deuil sont à toute fin pratique disparus du paysage des rites contemporains. [Thèse 4 , invariant 3]

- Avec la postmodernité, les rites funéraires ne concernent plus, le plus souvent, la collectivité, mais des microgroupes réunis autour de la connaissance immédiate du défunt. [Thèse 4, invariant 3]

- Avec la postmodernité, les grandes fonctions usuelles assumées par les rites funéraires jusqu'à la modernité ne sont plus l'apanage des seuls rites. [Thèse 4 , invariant 3]

- Avec la postmodernité, les rites funéraires ont un moindre impact thérapeutique. [Thèse 4, invariant 4]

- Avec la postmodernité, les rites funéraires ont tendance à se limiter à constater le décès ou la disparition d'un individu et à lui rendre un dernier hommage. [Thèse 4 , invariant 5 ]

- Avec la postmodernité, les croyances ou idéologies eschatologiques se diversifient et se multiplient. [Thèse 4, invariant 6 ]

- Avec la postmodernité, la diversification des idéologies eschatologiques contribue au développement des rites funéraires de proximité. [Thèse 4 , invariant 6]

- Avec la postmodernité, certains modes traditionnels de conservation des traces de nos morts s'estompent, alors que d'autres s'inventent. [Thèse 4, invariant 7]

- Avec la postmodernité, les rites funéraires parviennent plus difficilement à provoquer un effet d'intensification des relations sociales. [Thèse 5]

- Avec la postmodernité, les rites funéraires et les symboles les accompagnant arrivent peu à provoquer un changement de registre mental ou une transformation du cadre social des participants au rite. [Thèse 6]

- Avec la postmodernité, les rites funéraires insistent sur la composante « individuation » et sur l'histoire personnelle du défunt, tout en mettant en sourdine la composante «socialisation ». [Thèse 7]

En relisant ces dix-sept propositions, on se rend compte que douze d'entre elles concernent directement la notion des nouveaux rites 


\section{NESS.VDLUME NUMENO 1,2007}

funéraires - que l'on a associée à la mutation culturelle de la postmodernité occidentale -, rites qui seraient, entre autres, plus intimes et davantage minimalistes. C'est cette relation supposée entre des transformations macrosociales et des transformations à l'échelle de la ritualité funéraire et de notre rapport à la mort qui mérite de toute évidence d'être explorée en profondeur. Plusieurs propositions convergent en effet dans cette direction, soulevant des questions à la fois importantes et étroitement imbriquées. L'exploration ici de cette thématique centrale pourra permettre par la suite des avancées sur les autres questions mises de côté pour l'instant, faute d'une connaissance suffisamment précise des dits nouveaux rites funéraires.

\section{Bibliographie}

Anati, Emmanuel, La religion des origines, Paris, Bayard, 1999.

Bacqué, Marie-Frédérique (dir.), Mourir aujourd'bui. Les nouveaux rites funéraires, Paris, Odile Jacob, 1997.

Balandier, Georges, "D'unc espérance à l'autre. L'émergence de l'homme amortel ", dans Frédéric I.enoir et Jean-Philippe de Tonnac (dir.), La mort et l'immortalité. Encyclopédie des savoirs et des croyances, Paris, Bayard, 2004, p. 871-887.

Baudry, Patrick, "Paradoxes contemporains. Nouveaux rapports anthropologiques à la mort ", dans Frédéric Lenoir et Jean-Philippe de Tonnac (dir.), La mort et I'inmortalité. Encyclopédie des savoirs et des croyances, Paris, Bayard, 2004, p. 893-909.

Baudry, Patrick, "La mise en scènc de l'invisible ", Frontières, Variations sur le rite, vol. $10, \mathrm{n}^{\prime \prime} 2$, hiver 1998 , p. 7-10.

Baudry, Patrick, "Le sens de la ritualité funéraire ", dans Marie-Frédérique Bacqué (dir.), Mourir aujourd'bui. Les nouveaux rites funéraires, Paris, Odile Jacob, 1997, p. 223-244.

Baudry, Patrick et Henri-Pictre Jeudy, Le deuil impossible, Paris, Éditions Eshel, 2001. Besanceney, Jean-Claude, "Évolution des rites catholiques du deuil et nouvelles pratiques rituelles", dans Marie-Frédérique Bacqué (dir.), Mourir aujourd'bui. Les nomvaux rites funéraires, Paris, Odile Jacob, 1997, p. 167-195.

Berger, Peter, Comprendre la sociologie, Paris, Éditions du Centurion, 1973.

Boisvert, Yves, "Sortir du nihilisme. Quand la dictature du moi sert de bouée", dans Yves Boisvert et Lawrence (Olivier (dir.), A chacun sa quête. Essais sur les nouveaux lisages de la transcendance, Sainte-Foy, Presses de l'Université du Québec, 2000.

Boisvert, Yves, "L'analyse postmoderniste au-delà de l'esthétisme discursif », dans Yves Boisvert (dir.), Postmodernité et sciences humaines, Montréal, F́ditions Liber, 1998, p. $177-190$.

Cazeneuve, Jean, "Rite», dans Encyclopedia Universalis, Paris, 1985. 
Chamboredon, Jean-Claude, "I a restauration de la mort. Objets scientifiques et phantasmes sociaux ", Actes de la recherche en sciences sociales, vol. 2, n"2, 1976, p. 78 87.

Des Aulniers, Luce, «Rites d'aujourd'hui et de toujours », Frontières, V'ariations surle rite, vol. $10, \mathrm{n}^{\prime \prime} 2$, hiver 1998 , p. 3-6.

Des Aulniers, Luce, Itinérances de la maladie grave. Le temps des nomades, Paris, Montréal, I.'Harmattan, 1997.

Des Aulniers, Luce, «Bruit du temps jusqu'à silencc de mort», dans Marie-Frédérique Bacqué (dir.), Mourir aujourd'bui. I.es nowveaux rites funéraires, Paris, Odile Jacob, 1997, p. 197-222.

DeSpelder, Jynne Ann et Albert Lee Strickland, The Last Dance, Mountain View, Mayfield Publishing Company, 1996.

Dortier, Jean-François (dir.), "L'origine des cultures", Les grands dossiers des sciences bumaines, $\mathrm{n}^{\prime \prime} 1$, décembre 2005/janvier 2006, p. 26-94.

Dortier, Jean-François (dir.), I.e dictionnaire des sciences bumaines, Auxerre, Sciences Humaines Éditions, 2004.

Dortier, Jean-François, $L$ 'bomme, cet étrange animal... Aux origines du langage, de la culture et de la pensée, Auxerre, Sciences Humaines Éditions, 2004.

Druet, Pierre-Philippe, Pourvivre sa mort, Ars Moriendi, Paris, Éditions Lethielleux, 1981.

Gaudin, Philippe, La mort. Ce qu'en disent les religions, Ivry-sur-Seine, Les éditions de l'Atelier, 2001.

Goetschel, Roland, «Tu retourneras à la poussière. Pratiques et rituels de la mort dans le judaïsme ", dans Frédéric L.enoir et Jean-Philippe de Tonnac (dir.), La mort et l'immortalité. Encyclopédie des savoirs et des croyances, Paris, Bayard, 2004, p. 347-355.

Guetny, Jean-Paul, "Mort unique et deuil collectif ", Études sur la mort, n" 123, Morts et deuils collectifs, 2003, p. 99-102.

Gruau, Maurice, L'bomme rituel. Antbropologie du rituel catbolique français, Paris, Métailié, 1999.

Hanus, Michel, "Paroles, pratiques, rites et rituels ", fitudes sur la mort, n" 114, Rites et rituels, 1998 , p. 5-16.

Harrisson, Robert, Les morts, Paris, Le Pommier, 2003.

Hervieu-Léger, Danic̀le, La religion pour mémoire, Paris, Cerf, 1993.

Hervieu-Wane, Fabrice, Une boussole pour la vie. Les nouveaux rites de passage, Paris, Albin Michel, 2005.

Hocart, Arthur Maurice, Au commencement était le rite. De l'origine des sociétés bumaines, Paris, Éditions La Découverte, 2005.

Hulin, Michel, "L'homme et son double. L'invention de l'au-delà ", dans Frédéric Lenoir et Jean-Philippe de Tonnac (dir.), La mort et l'immortalité. Encyclopédie des savoirs et des croyances, Paris, Bayard, 2004, p. 53-78.

Javeau, Claude, «Retour sur les rites piaculaires : pratiques et rôles dans l'immédiat et à distance ", Études sur la mort, n"123, Morts et deuils collectifs, 2003, p. 69-78.

Jeffrey, Denis, Éloge des rituels, Québec, Presses de l'U niversité Laval, 2003.

Jeffrey, Denis, Jouissance du sacré, Paris, Armand Colin, 1998.

Jeffrey, Denis, "Éthique et postmodernité ", dans Yves Boisvert (dir.), Postmodernité et sciences humaines, Montréal, Liber, 1998, p. 137-160. 
Joncheray, Jean, "Rites et construction de l'identité : des rites de passage aux rites d'interaction et aux grands rites collectifs séculiers », dans Bernard Kaempf (dir.), Rites et ritualités, Paris, Les Éditions du Cerf, Lumen Vitae et Novalis, 2000, p. 393-414.

Laflamme, Simon et Ali Reguigui, Homogénéité et distinction, Sudbury, Prise de parole, 2003.

Lambert, Yves, Les valeurs des Français. Évolution de 1980 à 2000, Paris, Armand Colin, 2000.

Lardellier, Pascal, Théorie du lien rituel. Anthropologie et Communication, Paris, L'Harmattan, 2003.

Larouche, Jean-Marc, «La mort et le mourir, d'hier à aujourd'hui », Religiologiques, n“ 4, automne 1991, p. 1-41.

Leloup, Jean-Yves et Marie de Hennezel, L'art de mourir. Traditions religieuses et spiritualité bumaniste face à la mort, Paris, Pocket, 1997.

Lemieux, Raymond et Jean-Paul Montminy (dir.), Le catholicisme québécois, Québec, Les Éditions de l'IQRC, Les Presses de l'Université Laval, 2000.

Lenoir, Frédéric et Jean-Philippe de Tonnac, "Introduction », La mort et l'immortalité. Encyclopédie des savoirs et des croyances, Paris, Bayard, 2004, p. 19-37.

Lipovetsky, Gilles, L'ère du vide, Paris, Gallimard, 1983.

Loisel, J.-P. et O. Martin, Les Français et le souvenir des morts, Paris, novembre 2000.

Maffesoli, Michel, "De la "postmédiévalité "à la postmodernité ", dans Yves Boisvert (dir.), Postmodernité et sciences bumaines, Montréal, Liber, 1998, p. 9-21.

Maisonneuve, Jean, Les conduites rituelles, Paris, PUF, [1988] 1999.

Maltaux, André, Lazare, Paris, Gallimard, 1974.

Ménard, Guy, Petit traité de la vraie religion à l'usage de ceux et celles qui soubaitent comprendre un peu mieux le vingt et unième siècle, Montréal, Liber, 1999.

Ménard, Guy, "Le bricolage des dieux. Pour une lecture postmoderniste du phénomène religieux", dans Yves Boisvert (dir.), Postmodernifé et sciences humaines, Montréal, Liber, 1998, p. 89-115.

Métraux, Jean-Claude, Deuils collectifs et création sociale, Paris, La dispute, 2004.

Mohen, Jean-Pierre, «Le propre de l'espèce humaine. Rites funéraires et destination du cadavre dans la préhistoire et les traditions anciennes ", dans Frédéric Lenoir et Jean-Philippe de Tonnac (dir.), La mort et l'immortalité. Encyclopédie des savoirs et des croyances, Paris, Bayard, 2004, p. 288-290.

Morin, Edgar, L'bomme et la mort, Paris, Le Seuil, [1951] 1970.

Rappaport, Roy A., Ritual and religion in the making of bumanity, Cambridge, Cambridge University Press, 1999.

Richard, Isabelle, "Mourir à l'hôpital ", dans Marie-Frédérique Bacqué (dir.), Mourir aujourd'bui. Les nouveaux rites funéraires, Paris, Odile Jacob, 1997, p. 119-147.

Rivière, Claude, Les rites profanes, Paris, PUF, 1995.

Segalen, Martine, Rites et rituels contemporains, Paris, Nathan, 1998.

St-Onge, Sébastien, L'industrie de la mort, Québec, Éditions Nota bene, 2001.

Taylor, Charles, J a diversité de l'expérience religieuse aujourd'bui, Montréal, Bellarmin, 2003.

Thomas, Louis-Vincent, La mort, Paris, PUF, [1988] 2003. 


\section{LUC BUSSIÈRES / RITES FUNĖBRES ET SCIENCES HUMAINES...}

Thomas, Louis-Vincent, Les chairs de la mort, Paris, Institut d'édition Sanofi-Synthélabo, 2000.

Thomas, Louis-Vincent, "Problemes actuels de la mort en Occident ", dans Prétentaine, Université de Montpelier 3, 1994, sous la section Hommage à Louis-Vincent Thomas et dont un extrait a été repris sous le titre «Louis-Vincent Thomas ou l'Occident revisité », Frontières, vol. 11, n" 1, 10" anniversaire, 1998, p. 15-20.

Thomas, Iouis-Vincent, "Nature de la mort", dans Luc Bessette (dir.), Lee processus de guérison : par-delà la souffrance ou la mort, Beauport (Qc), MNH, 1994, p. 206-220.

Thomas, Louis-Vincent, «La mort aujourd'hui : de l'esquive au discours convenu », Religiologiques, n" 4, automne 1991, p. 1-32.

Thomas, Louis-Vincent, Rites de mort. Pour la paix des vivants, Paris, Fayard, 1985.

Thomas, Louis-Vincent, Mort et pouvoir, Paris, Payot, 1978.

Turner, Victor, Le phénomène rituel. Structure et contre-structure, Paris, PUF, 1990.

Turner, Victor, From ritual to theatre, New York, Performing Arts Journal Publications, 1982.

Urbain, Jean-Didier, L'arbhipel des morts. Le sentiment de la mort et les dérives de la mémoire dans les cimetières d'Occident, Paris, Éditions Payot et Rivages, [19891 1998.

Urbain, Jean-Didier, "L.a cendre ct la trace. La vogue de la crémation ", Frédéric I.enoir et Jean-Philippe de Tonnac (dir.), La mort et l'immortalité. Encyclopédie de la mort et des croyances, Paris, Bayard, 2004, p. 1207-1217.

Van Gennep, Arnold, Les rites de passage, Paris, Picard, [1909] 1981.

Volant, Éric, “La religion et la mort », dans Jean-Marc Larouche et Guy Ménard (dir.), L'étude de la religion au Québec. Bilan et prospective, Québec, Les Presses de l'Université I aval/Corporation canadienne des sciences religieuses, 2001, p. 323.

Volant, Éric, Jeux mortels et enjeux éthiques, Chicoutimi, Sapienta, 1992.

Vovelle, Michel, La mort et l'Occident de 1300 à nos jours, Paris, Gallimard, 1983. 